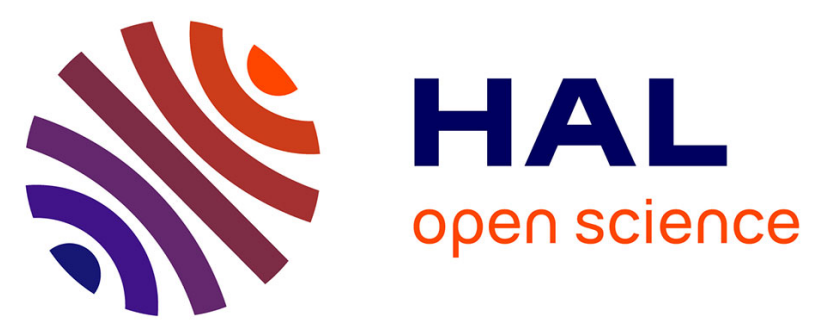

\title{
A Millimeter-wave Interferometric Study of Dust and CO Disks Around Late Spectral Type Stars in Taurus-Auriga
}

\author{
G. H. Schaefer, Anne Dutrey, S. Guilloteau, M. Simon, R. J. White
}

\section{To cite this version:}

G. H. Schaefer, Anne Dutrey, S. Guilloteau, M. Simon, R. J. White. A Millimeter-wave Interferometric Study of Dust and CO Disks Around Late Spectral Type Stars in Taurus-Auriga. The Astrophysical Journal, 2009, 701 (1), pp.698-709. 10.1088/0004-637X/701/1/698 • hal-00417575

\section{HAL Id: hal-00417575 \\ https://hal.science/hal-00417575}

Submitted on 16 Sep 2009

HAL is a multi-disciplinary open access archive for the deposit and dissemination of scientific research documents, whether they are published or not. The documents may come from teaching and research institutions in France or abroad, or from public or private research centers.
L'archive ouverte pluridisciplinaire HAL, est destinée au dépôt et à la diffusion de documents scientifiques de niveau recherche, publiés ou non, émanant des établissements d'enseignement et de recherche français ou étrangers, des laboratoires publics ou privés. 


\title{
A Millimeter Wave Interferometric Study of Dust and CO Disks Around Late Spectral Type Stars in Taurus-Auriga
}

\author{
G. H. Schaefer ${ }^{1}$, A. Dutrey ${ }^{2,3}$, S. Guilloteau ${ }^{2,3}$, M. Simon ${ }^{4}$, and R. J. White ${ }^{5,6}$
}

\begin{abstract}
We present a new millimeter survey of 23 pre-main-sequence stars in the Taurus-Auriga star forming region. The main goal of the project was to identify circumstellar disks around stars of late spectral types $\left(M_{*} \lesssim 0.7 \mathrm{M}_{\odot}\right)$. We used the IRAM Plateau de Bure Interferometer to obtain observations at $1.3 \mathrm{~mm}$, $2.7 \mathrm{~mm}$, and the $\mathrm{CO} \mathrm{J}=1-0$ and $\mathrm{J}=2-1$ lines. We detected eight sources in continuum emission, with new detections of CIDA-1 and CIDA-8 at $1.3 \mathrm{~mm}$. Overall, we find that circumstellar disks around stars with spectral types later than M2 tend to have smaller millimeter continuum fluxes than those around earlier type stars. We also present four detections of disks in their CO line emission ( $\mathrm{LkH} \alpha 358$, GO Tau, Haro 6-13, IRAS 04385+2550). By mapping the rotation of the resolved $\mathrm{CO}$ emission from the circumstellar disks, we obtain estimates of the dynamical mass of the central stars.
\end{abstract}

Subject headings: circumstellar matter — radio lines: stars — radio continuum: stars - stars: pre-main sequence — stars: fundamental parameters

\section{Introduction}

Determining the masses of pre-main-sequence (PMS) stars is important for understanding the physical processes of star formation. Usually this is accomplished by comparing the

\footnotetext{
${ }^{1}$ The CHARA Array of Georgia State University, Mount Wilson Observatory, Mount Wilson, CA 91023; schaefer@chara-array.org

${ }^{2}$ Université Bordeaux 1, Laboratoire d'Astrophysique de Bordeaux (LAB)

${ }^{3} \mathrm{CNRS} / \mathrm{INSU}$ - UMR 5804, BP 89, 33270 Floirac, France

${ }^{4}$ Department of Physics and Astronomy, Stony Brook University, Stony Brook, NY 11794-3800

${ }^{5}$ Department of Physics, University of Alabama in Huntsville, Huntsville, AL 34899

${ }^{6}$ Department of Physics and Astronomy, Georgia State University, Atlanta, GA 30303
} 
locations of stars on an H-R diagram to theoretical tracks of pre-main sequence (PMS) evolution. However, the wide variety of available calculations, each with their own assumed physics, predict a large range of possible stellar masses at young, PMS ages (e.g. Simon 2001; Hillenbrand \& White 2004; Mathieu et al. 2007). A powerful way to distinguish among these calculations, and thereby test the input physics, is to compare predicted masses to masses obtained by dynamical methods.

Binary stars are one of the most effective tools for determining accurate dynamical masses (e.g. Andersen 1991). These systems often permit precise measurements of stellar mass ( $\sim 1 \%$ accuracy), although this is often only achieved after years of observations. Moreover, the constraint they provide on evolutionary models is sometimes limited by poorly determined stellar properties because the two stars are often difficult to observe separately. This is especially problematic for young binaries, which are usually at large (> $100 \mathrm{pc})$ distances and more challenging to resolve spatially.

Mapping the rotational velocity of a spatially and spectroscopically resolved circumstellar disk provides a unique way to measure the dynamical mass of a young single star in just one observational epoch. One successfully demonstrated way to do this is by measuring the CO emission line velocities using an array of millimeter antennas that can spatially resolve the star's circumstellar disk (e.g. Guilloteau \& Dutrey 1998; Simon et al. 2000). Using this technique, dynamical mass precisions of 3\% have been achieved (e.g. Piétu et al. 2007).

The goal of the present study is to increase the number of young low-mass single stars with dynamical masses measured by mapping the rotation of their circumstellar disks using the Plateau de Bure Interferometer at IRAM. We focus specifically on low-mass stars because until just recently (Stassun et al. 2006; Irwin et al. 2007), there were no PMS stars below $0.5 \mathrm{M}_{\odot}$ with dynamically determined masses at a precision better than $10 \%$, a level necessary to distinguish among the evolutionary models. In Section 2 we describe the properties of our sample and summarize the Plateau de Bure observations, and in Section 3 we present the observational results. In Section 4 we calculate fundamental disk properties of the four stars with detected CO line emission; this analysis also yields dynamical masses for these four stars. In Section 5 we use our new measurements, in combination with previous work, to investigate the stellar mass dependencies of circumstellar disk properties and to test the predictions of theoretical evolutionary models. We summarize our findings in Section 6 . 


\section{Sample of Stars and Observations}

\subsection{Sample Selection}

Only confirmed members of the Taurus star forming region were observed in this study. At the time this program began (2001), recent discoveries were extending the well studied stellar population of Taurus (e.g. Kenyon \& Hartmann 1995) to much lower stellar masses, including brown dwarfs (Briceño et al. 1998, 1999; Martín 2000). From this newly expanded membership list, we selected an initial sample consisting of all stars with spectral types of M3 and later that had no known companions identified in high-resolution imaging studies (e.g. Simon et al. 1995; White \& Ghez 2001, and references therein), and that were classified as classical T Tauri stars based on the strength of $\mathrm{H} \alpha$ emission (e.g. White \& Basri 2003). The restriction to cool spectral types yielded a sample with estimated masses $\lesssim 0.5 \mathrm{M}_{\odot}$. Single stars were selected since the stellar and disk properties are much easier to interpret and classical T Tauri stars were selected because these stars have disks detectable at millimeter wavelengths $53 \%$ of the time, compared to $29 \%$ for weak-lined T Tauri stars (Beckwith et al. 1990, statistics are based on T Tauri stars with spectral types $\sim$ K5 - M3, however).

Twelve stars met these criteria, and to this sample we also added the two "continuum" stars CIDA-1 and GM Tau (Briceño et al. 1993); spectra of these two stars that were subsequently published in White \& Basri (2003) indicated that they had mid-M spectral types as well. We note that three of these 14 stars had previously been surveyed for $1.3 \mathrm{~mm}$ continuum emission (Beckwith et al. 1990); two were detected (FN Tau and LkH $\alpha$ 358) while the third star (FP Tau) had an anomalously large upper limit of $50 \mathrm{mJy}$.

To begin assessing the possibility of determining dynamical masses from the circumstellar material orbiting these stars, all but the two stars with previous detections at millimeter wavelengths were observed with the IRAM interferometer (Section 2.2). Unfortunately, we detected only two of these 12 stars in continuum emission and none in CO spectral line emission. We speculated that this could be caused by a decrease in millimeter emission from lower mass stars. Therefore we relaxed the spectral type restriction to include all single, classical $\mathrm{T}$ Tauri stars with an $\mathrm{M}$ spectral type (out to spectral type M7) in the subsequent observations. Thus, in addition to FN Tau and $\mathrm{LkH} \alpha 358$ noted above, this added six stars that Beckwith et al. (1990) detected $1.3 \mathrm{~mm}$ emission from (GO Tau, FT Tau, Haro 6-13, DH Tau, DN Tau and IQ Tau) and five stars that had not been surveyed for $1.3 \mathrm{~mm}$ emission (CIDA-7, IRAS 04108+2910, IRAS 04108+2803A, IRAS 04216+2603, and IRAS 04385+2550; Briceño et al. 1993; Kenyon et al. 1998), bringing the total number of stars in the sample to 25. Due to a typographical error, Kenyon et al. (1998) mislabel IRAS $04108+2803$ A as IRAS $04108+2805$, which is not an IRAS source (S. Kenyon, priv. 
comm.). Originally, LkH $\alpha 358$ was classified as an M5.5 star (Cohen \& Kuhi 1979; Kenyon et al. 1998), however, recent high resolution spectra indicate a spectral type of K7-M0 (Muzerolle et al. 2003); a discussion of this discrepancy and an additional spectrum of this star, which is consistent with the hotter spectral type, is presented in the Appendix. Of the 13 additional M-stars, we observed 10 (due to time constraints) and also included earlier IRAM observations of GO Tau obtained by T. Forveille and collaborators. The full set of observations (M0-M7) yielded a total of eight detections of disks in continuum emission but only four in their CO lines.

The observed sample of 23 stars, in combination with previous millimeter observations of Taurus stars, establishes a complete millimeter survey of single M0-M7 classical T Tauri stars that were known at the time this program began. Table 1 provides the essential data for these 23 stars ordered by spectral type, listed in Column 2. Column 3 provides the reference for the spectral type. Stellar luminosities calculated by Kenyon \& Hartmann (1995, $L_{\mathrm{J}}$ values in their paper) are listed in column 4 and stellar luminosities calculated by White \& Ghez (2001) or White \& Hillenbrand (2004) are listed in column 5; we assemble both sets of values for comparison and completeness. Since several stars do not have stellar luminosity estimates, we also assemble bolometric luminosities from Briceño et al. $(1998,1999,2002)$ in column 6 and from Luhman $(2000,2004)$ in column 7. All of the luminosities are evaluated for a distance of 140pc (Kenyon et al. 1994). The effective temperatures, $T_{\text {eff }}$ in column 8 , are estimated using the dwarf spectral type-temperature relations used in Kraus \& Hillenbrand (2007) for K7-M0 and the T-Tauri temperature scale defined in Luhman et al. (2003b) for M1-M7. Column 9 lists the dates of our millimeter-wave observations.

\subsection{IRAM Interferometer Observations}

The 22 late spectral type stars in our main program were observed with the IRAM interferometer in the D configuration on 2001 November 21, 2002 April 15, 2002 April 17, and 2002 October 4. Each source was observed in the ${ }^{12} \mathrm{CO} \mathrm{J}=1-0(115.3 \mathrm{GHz})$ and $\mathrm{J}=2-1$ $(230.5 \mathrm{GHz})$ lines and in the continuum at 2.7 and $1.3 \mathrm{~mm}$. The correlator was set up to have one band of $20 \mathrm{MHz}$ centered on the ${ }^{12} \mathrm{CO} \mathrm{J}=1-0$ line, one band of $20 \mathrm{MHz}$ centered on the ${ }^{12} \mathrm{CO} \mathrm{J}=2-1$ line, and two bands of $320 \mathrm{MHz}$ for both the 1.3 and $2.7 \mathrm{~mm}$ continuum. The spectral resolutions were 0.23 and $0.12 \mathrm{~km} \mathrm{~s}^{-1}$ in the 2.7 and $1.3 \mathrm{~mm}$ narrow bands, respectively.

The three sources with the strongest CO emission $(\mathrm{LkH} \alpha 358$, IRAS $04385+2550$, and Haro 6-13) were followed up with observations during one transit in the $\mathrm{C}$ configuration on 2002 December 20. Baselines ranging from 24 to 160 meters in the C and D configu- 
rations provided an angular resolution of $1.4^{\prime \prime} \times 0.8^{\prime \prime}$ at $\mathrm{PA} 37^{\circ}$ for the $1.3 \mathrm{~mm}$ continuum and $2.8^{\prime \prime} \times 1.8^{\prime \prime}$ at PA $35^{\circ}$ for the $2.7 \mathrm{~mm}$ continuum. For both projects, the phase and amplitude calibrators observed during the observations were $0415+379$ and $0528+134$. The flux calibration was performed using MWC349, 3C273, or 3C454.3 (see IRAM flux report 14 , for details). Good weather conditions yielded phase errors not higher than $\sim 25^{\circ}$ and $\sim 50^{\circ} \mathrm{rms}$ at 2.7 and $1.3 \mathrm{~mm}$, respectively. All of the data were reduced using the GILDAS software package.

GO Tau was observed in the D configuration on 1995 November 22, 1995 December 5, and 1997 April 5 by T. Forveille and collaborators. The amplitude and phase calibrators used were 3C 111 and $0528+134$. GO Tau was observed in the ${ }^{12} \mathrm{CO} \mathrm{J}=1-0(115.3 \mathrm{GHz})$ and $\mathrm{J}=2-1(230.5 \mathrm{GHz})$ lines and in the continuum at 2.7 and $1.3 \mathrm{~mm}$. The correlator was set up to have one band of $20 \mathrm{MHz}$ centered on the ${ }^{12} \mathrm{CO} \mathrm{J}=1-0$ line, one band of $20 \mathrm{MHz}$ centered on the ${ }^{12} \mathrm{CO} \mathrm{J}=2-1$ line, and two bands of $160 \mathrm{MHz}$ for both the 1.3 and $2.7 \mathrm{~mm}$ continuum.

\section{Results}

\subsection{Millimeter Continuum Results}

In Table 2 we present the observed $2.7 \mathrm{~mm}$ and $1.3 \mathrm{~mm}$ flux densities. For the sources which were not detected, we provide $3 \sigma$ upper limits. The flux densities and positions were determined in the $u v$ plane by fitting a circular Gaussian to the data with a full width at half maximum of $0.3^{\prime \prime}$, set by the estimated seeing. We followed this procedure because most of the detected sources are not resolved and hence have disks with apparent sizes $<0.3^{\prime \prime}$ or $\sim 42 \mathrm{AU}$ at the distance of the Taurus star forming region. Evidently, most of the dust in the disks of the stars in this study is concentrated at smaller radii than the more massive stars studied by Simon et al. (2000) and Piétu et al. (2006). In Table 2 we also list other $1.3 \mathrm{~mm}$ and $850 \mu \mathrm{m}$ measurements reported in the literature (Beckwith et al. 1990; Osterloh \& Beckwith 1995; Motte \& André 2001; Andrews \& Williams 2005, 2007). Our results agree with these values within 1-2 $\sigma$. In the last column, we give the spectral index $\alpha$, in the

$\mathrm{F}_{\nu} \propto \nu^{\alpha}$ sense, calculated through a least-squares fit using all of the available values listed in Table 2 .

Table 3 lists the centroid coordinates of the $1.3 \mathrm{~mm}$ continuum emission (except for IQ Tau for which we used the coordinates based on the $2.7 \mathrm{~mm}$ emission). The astrometric precision for each target is given in the last column of Table 3. The coordinates agree with the values given in the 2MASS catalog (Cutri et al. 2003; Skrutskie et al. 2006) within $0.1-1.8^{\prime \prime}$. 
We derived the physical properties of the dust disks based on the 1.3 and $2.7 \mathrm{~mm}$ continuum emission. We modeled the disk in the Fourier plane using power law distributions for the temperature and surface density (e.g. Dutrey et al. 1994, 1996) assuming $T_{\text {dust }}=15 \mathrm{~K}$ at $100 \mathrm{AU}$, a temperature exponent of $q=0.3$, and a surface density exponent of $p=1.5$. The derived values for the outer radius and mass of the dust disk are given in Table 4 . The disk masses are based on the Beckwith et al. (1990) prescription assuming a dust emissivity index $\beta=1$ and are uncertain by at least a factor 2 . For the weaker, unresolved sources, we quote a lower limit to the disk radius (assuming optically thick emission) and a lower limit to the disk mass (assuming optically thin emission) using constant $T_{\text {dust }}=15 \mathrm{~K}$.

\subsection{CO Line Emission}

We centered the CO line maps on the continuum coordinates given in Table 3 and applied natural weighting to the visibilities. Figures $1-4$ show the ${ }^{12} \mathrm{CO}$ line maps for GO Tau, IRAS $04385+2550, \mathrm{LkH} \alpha 358$, and Haro 6-13. For $\mathrm{LkH} \alpha 358$ we show only the ${ }^{12} \mathrm{CO}$ $\mathrm{J}=2-1$ line map because the resolution and $\mathrm{S} / \mathrm{N}$ was insufficient to construct a map in the $\mathrm{J}=1-0$ line.

The ${ }^{12} \mathrm{CO}$ line maps for IRAS $04385+2550, \mathrm{LkH} \alpha 358$, and Haro 6-13 show an absence of emission in the central velocity channels. For GO Tau, only the blue-shifted emission from the disk is detected. The lack of emission in these channels is caused by the molecular cloud in which these stars are located. Because the cloud is optically thick in ${ }^{12} \mathrm{CO}$ it obscures the channels near the systemic velocities (or at the red-shifted velocities for GO Tau). We omitted these obscured velocity channels from our analysis in $\S 4$. Away from the masking effects of the molecular cloud, the CO line emission of GO Tau, IRAS $04385+2550$, and $\mathrm{LkH} \alpha 358$ indicates an origin in an extended rotating disk.

\section{Analysis}

The CO line data were modeled as a rotating disk using the $\chi^{2}$ minimization procedure originally developed by Guilloteau \& Dutrey (1998) and recently optimized by Piétu et al. (2007). We fit the data using cylindrical coordinates and by assuming a hydrostatic scale height. As discussed by Piétu et al. (2007), the accuracy of the dynamical mass does not depend on either of these choices. We removed the velocity channels obscured by the molecular cloud from the $\chi^{2}$ analysis. The good channels that we used are indicated by the fiducial crosses that mark the map centers in Figures 1-4. When applicable, we performed a 
simultaneous fit to the ${ }^{12} \mathrm{CO} \mathrm{J}=1-0$ and $\mathrm{J}=2-1$ transitions (see Piétu et al. 2007, for details). This improves the accuracy by almost $\sqrt{2}$ compared to fitting the transitions separately.

Table 5 lists the geometric and kinematic disk parameters determined for the sources where CO was detected: $\mathrm{LkH} \alpha 358$, GO Tau, Haro 6-13, and IRAS 04385+2550. The parameters include the orientation (P.A.) and inclination $(i)$ derived from the $\mathrm{CO}$ emission (except for Haro 6-13, the disks are not significantly resolved in continuum), the outer radius $\left(R_{\text {out }}\right)$, the projected velocity at $100 \mathrm{AU}\left(V_{100} \sin i\right)$, and the velocity exponent $v$ of the radial power law $V(r)=V_{100}(r / 100 \mathrm{AU})^{-v}$. For all sources, we find $v=0.5$, consistent with Keplerian rotation, which allows us to derive the dynamical mass of the central star from

$$
M=\left[\frac{\left(V_{100} \sin i\right)}{2.98 \sin i}\right]^{2} M_{\odot}
$$

where $2.98 \mathrm{~km} \mathrm{~s}^{-1}$ is the circular velocity at a radius of $100 \mathrm{AU}$ for a $1 M_{\odot}$ star (Simon et al. 2000). Since the rotation velocity and the inclination are tightly coupled (Guilloteau \& Dutrey 1998), the formula includes the two measured quantities, $V_{100} \sin i$ and $i$. The derived masses scale as $M \propto V^{2}(r) r$ and, hence, depend linearly on the distance to the star. In computing the outer radii and dynamical masses, we used the average distance to the Taurus star forming region of $140 \pm 10$ pc (Kenyon et al. 1994). Since the distance can be improved through techniques independent of the mass measurement, this uncertainty is not propagated to the masses in Table 5.

In a Keplerian disk, the inclination is largely determined by the shape of the emission as a function of velocity near the systemic velocity. Therefore, the obscuration of the central velocity channels limits the precision of the measured inclinations and, hence, the dynamical masses.

Table 6 lists the physical parameters of the disk derived from the fit to the CO data. These parameters include the temperature at $100 \mathrm{AU}\left(T_{100}\right)$, the temperature radial exponent $q$ of the temperature power law $T(r)=T_{100}(r / 100 \mathrm{AU})^{-q}$, the scale height at $100 \mathrm{AU}$ $\left(H_{100}\right)$, and the radial exponent $h$ of the scale height power law $H(r)=H_{100}(r / 100 \mathrm{AU})^{-h}$. For all of the sources, we show the temperature and scale heights derived from assuming hydrostatic equilibrium (columns 2-5). For GO Tau and IRAS 04385+2550, the two sources where we have the most complete CO data, we also show the temperature, scale height, and corresponding exponents fitted independently with power laws for comparison (columns 6-9). 


\subsection{Comments on Individual Objects}

GO Tau: Because of obscuration from the molecular cloud, only the blue-shifted emission from the disk of GO Tau is detected (see Figure 1). We derived the disk parameters from a simultaneous fit to the ${ }^{12} \mathrm{CO} \mathrm{J}=1-0$ and $\mathrm{J}=2-1$ transitions. The velocity exponent is in good agreement with Keplerian rotation. The large CO disk of GO Tau is well-resolved by the interferometer observations and provides an accurate dynamical map. Combined with the presence of strong disk emission near the systemic velocity, this leads to a precise determination of the inclination which yields to a tightly constrained dynamical mass $0.61 \pm$ $0.02 \mathrm{M}_{\odot}$ for the central star at a fiducial distance of $140 \mathrm{pc}$. The internal precision of $3.3 \%$ is quite impressive, however, including the uncertainty in the distance to individual stars in the Taurus star forming region (see Sect 4 and 5.2) adds another $10 \%$ to the error budget.

IRAS 04385+2550 (Haro 6-33): The ${ }^{12} \mathrm{CO}$ line maps for IRAS 04385+2550 show an absence of emission in the central velocity channels (see Figure 2). We performed a simultaneous fit to the ${ }^{12} \mathrm{CO} \mathrm{J}=1-0$ and $\mathrm{J}=2-1$ transitions to determine the disk parameters. The velocity exponent is consistent with Keplerian rotation. The CO disk of IRAS $04385+2550$ is reasonably well-resolved, but the precision of the inclination is limited by the obscuration of the central velocity channels. In turn, this affects the precision of the dynamical mass estimate, for which we determine a value of $0.5 \pm 0.1 \mathrm{M}_{\odot}$ at a fiducial distance of $140 \mathrm{pc}$.

LkH $\alpha$ 358: The maps for $\mathrm{LkH} \alpha 358$ show a striking absence of emission in the central velocity channels (see Figure 3 ). Unfortunately the ${ }^{12} \mathrm{CO} \mathrm{J}=1-0$ line observations have insufficient resolution and $\mathrm{S} / \mathrm{N}$ to construct a dynamical model, so the derived disk parameters are solely from a fit to the ${ }^{12} \mathrm{CO} \mathrm{J}=2-1$ line observations. Because of a lack of good spatial resolution across the small $\mathrm{CO}$ disk of $\mathrm{LkH} \alpha 358$, we fixed the velocity and temperature exponents to 0.5. However, even after adopting these disk parameters, the poorly constrained inclination yields only an imprecise dynamical mass that is of little value. The mass range given for $\mathrm{LkH} \alpha 358$ in Table $5\left(0.5-2.0 \mathrm{M}_{\odot}\right)$ represents the broad $1 \sigma$ uncertainty interval.

Haro 6-13: The ${ }^{12} \mathrm{CO}$ line maps for Haro 6-13 show an absence of emission in the central velocity channels as well as evidence of extended emission and high velocity wings that could be associated with an outflow (see Figure 4). Although the strong continuum emission of Haro 6-13 indicates a dusty environment, it is unclear whether the extended CO emission can be interpreted in terms of a rotating disk. We attempted to fit the $\mathrm{CO}$ emission as a rotating disk by computing a simultaneous fit of the $\mathrm{J}=1-0$ and $\mathrm{J}=2-1$ transitions. However, only a few outer channels could be used because of the obscuration. We fixed the disk 
position at the center determined from the continuum images. The orientation derived from the CO maps and the continuum are consistent, giving confidence in the derived values. The velocity exponent agrees with Keplerian rotation. However, only a lower limit on the disk radius could be measured, since all of the low velocity channels are unusable because of the confusion with the molecular cloud. The removal of all but the outer velocity channels also severely limits the accuracy of the inclination. As a result, the derived mass of $1.0 \pm 0.15 \mathrm{M}_{\odot}$ likely suffers from a systematic bias which the statistical error does not take into account (see discussion in Sect 5.2).

\section{Discussion}

\subsection{Mass Dependent Disk Properties}

The motivation for this survey was to increase the number of low mass stars with accurate dynamically determined stellar masses from mapping the disk kinematics. Unfortunately, our results indicate that it is difficult to detect CO disks around late-type low-mass stars given the current detection sensitivities. Of the 23 stars surveyed here, which have spectral types roughly uniformly distributed between K7 and M7 (Table 1), only four CO disks were detected (17\%). Of the four stars with detected CO disks, all four have spectral types of M0.5 or hotter. Thus far, the latest spectral type of a star in the Taurus-Auriga star forming region for which millimeter CO emission has been detected is M1 (DM Tau and CY Tau; e.g. Simon et al. 2000; Piétu et al. 2007).

Figure 5 shows a plot of the radii of CO disks detected around T Tauri stars of spectral type K-M in Taurus Auriga (Dutrey et al. 1994; Koerner \& Sargent 1995; Duvert et al. 1998; Simon et al. 2000; Yokogawa et al. 2002; Dutrey et al. 2003; Pety et al. 2006; Piétu et al. 2007; Dutrey et al. 2008; Guilloteau et al. 2008). We chose to plot the radius because it was a common property measured in all of the cited references, however we note the difficulty in determining the disk outer radius when confusion with the molecular cloud exists. In the figure, we also distinguish between circumstellar and circumbinary disks. While the sample size is too small to comment on correlations between the size of the gas disk and spectral type of the star, the lack of detected disks in CO emission for spectral types of M2 and later is quite apparent. The absence of detected CO disks around these later-type stars is partially due to the sensitivity of the observations. We speculate that the temperature of such disks should not be very different from "normal" T Tauri disks, unless sedimentation of dust becomes more important. Heating by cosmic rays and the ambient interstellar radiation

field sets a lower limit of about $7 \mathrm{~K}$ for the disk temperature. Given this, the scale height will be somewhat larger, but only as a function of $1 / \sqrt{M}$ (e.g. Dutrey et al. 1994). Similarly, 
the outer radius will be smaller, since it is roughly defined by where the thermal velocity is equal to the rotational velocity $(H / R=1)$. Again, this is a $\sqrt{M}$ effect. More flaring may result, causing the gas to be heated and making the disk radius even smaller $(\propto 1 / \sqrt{T})$. Additionally, photo-dissociation, especially by the ambient UV field will destroy CO more easily in these disks. Overall, the combined effect of these considerations makes it more difficult to detect $\mathrm{CO}$ gas disks around the lowest mass stars.

Our results also indicate that it is difficult to detect continuum emission from dusty disks around late-type low-mass stars. To investigate this further, we combined our results with the $1.3 \mathrm{~mm}$ surveys of the Taurus-Auriga star forming region by Beckwith et al. (1990), Osterloh \& Beckwith (1995), Motte \& André (2001), and Scholz et al. (2006). We counted all detections that were less than $3 \sigma$ results as nondetections. We removed redundancies between the lists by selecting the values of Osterloh \& Beckwith (1995), mostly because these measurements tended to be more precise and the upper limits were a factor of two better than in Beckwith et al. (1990). We obtained spectral types for the sample from the compilation of Kenyon \& Hartmann (1995), with updates of more recent measurements from Briceño et al. (1993); Hartigan et al. (1994); Kenyon et al. (1998); Martín (2001); White \& Ghez (2001); Briceño et al. (2002); Hartigan \& Kenyon (2003); Luhman et al. (2003a); White \& Basri (2003); Luhman (2004); White \& Hillenbrand (2004). Using this combined sample, we computed $1.3 \mathrm{~mm}$ detection rates for different spectral ranges. We decided to choose M3 as the division line because of the apparent break in the strength of the continuum emission at this spectral type. For stars with spectral types between K0-M2, we find 50 out of 110 $(0.46 \pm 0.08)$ have detectable dust disks. For stars with spectral types of M3 and later only 11 out of $56(0.20 \pm 0.07)$ have detectable disks. Thus, the more massive stars are more likely to have detectable disks, a difference significant at about the $2.3 \sigma$ level.

To examine how the $1.3 \mathrm{~mm}$ emission depends upon spectral type, in Figure 6 we plot the $1.3 \mathrm{~mm}$ flux density versus spectral type for the K0-M9 stars in the sample compiled from Beckwith et al. (1990), Osterloh \& Beckwith (1995), Motte \& André (2001), Scholz et al. (2006), and our sample of 23 low-mass PMS stars. The squares indicate the detections while the triangles represent the $3 \sigma$ upper limits. There appears to be a decrease in the strength of the $1.3 \mathrm{~mm}$ flux densities for stars of spectral type M3 and later, corresponding to roughly $M<0.5 \mathrm{M}_{\odot}$ for the Baraffe et al. (1998) evolutionary tracks. The top panel of Figure 7 shows the cumulative distributions of continuum emission strength for stars with spectral types between K0-M2 and M3-M9. While the general shape of the two distributions are similar, the higher mass stars consistently have higher $1.3 \mathrm{~mm}$ flux densities. A KolmogorovSmirnov test suggest that the two distributions are significantly different, with a negligible probability $(p \ll 0.01)$ that this occurred by chance. 
The declining disk detection rate and flux density with spectral type suggest that circumstellar disk masses are decreasing with spectral type. In Figure 8 we plot the eight disk masses or lower limits listed in Table 4, along with 128 disk masses from Andrews \& Williams (2005) and Scholz et al. (2006) with spectral type determinations taken from our compiled list of references described above. There indeed seems to be a lack of massive disks around stars with spectral types of M3 and later. We note that for stars of spectral types M2 and earlier, the range of values found for the disk masses increases. Specifically, the earlier type stars $\left(M>0.5 M_{\odot}\right)$ tend to have both large and small disks while the later type stars $\left(M<0.5 M_{\odot}\right)$ show an absence of the most massive disks. The bottom panel of Figure 7 shows the cumulative distributions of dust mass for stars with spectral types between K0-M2 and M3-M9. A Kolmogorov-Smirnov test indicates a negligible probability $(p \ll 0.01)$ that the two distributions are the same. These results agree with the conclusions of Scholz et al. (2006) who find that the ratio between disk mass and stellar mass does not change, implying that more massive stars have more massive disks. In our analysis, we have used spectral type as a tracer of mass. As mentioned by Scholz et al. (2006), these results are contrary to Andrews \& Williams (2005) who did not find any correlations between the submillimeter properties and the mass, effective temperature, or luminosity of the star. The data that we plot in Figure 8 is essentially the same as Andrews \& Williams (2005), except that we focus on a narrower spectral range of K-M stars and include more late-type stars (our sample and those of Scholz et al. 2006), whereas Andrews \& Williams (2005) use stars across the wider spectral range of A0-M5. This might partially explain the discrepancy in the results. However, Scholz et al. (2006) based their findings on an age-selected sample and suggest that discrepancies with other results might be caused by an age spread or other environmental effects on the disks.

The presence of a binary companion also effects the distribution of disk masses (e.g. Osterloh \& Beckwith 1995; Scholz et al. 2006). For the continuum detections in Figures 6 and 8, we identify the known binaries as filled circles while the single stars are plotted as filled squares (Ghez et al. 1993; Simon et al. 1995; White \& Ghez 2001; Duchêne et al. 2004, 2007; Kraus et al. 2006; Konopacky et al. 2007; Connelley et al. 2008). We note that several of the strongest $1.3 \mathrm{~mm}$ continuum sources are found in binary systems. This is expected from momentum balance; the higher the angular momentum, the larger the disk, and the more likely the formation of a binary (or multiple) system. This may also be the case for the strongest source in the M3-M9 range (IRAS 04158+2805). Andrews et al. (2008) have recently mapped the disk of IRAS $04158+2805$ in the CO $\mathrm{J}=3-2$ transition and found that the dynamical mass of the central star determined from the disk rotation exceeds the predicted evolutionary mass by a factor of two and speculate that the central source might be an equal-mass binary. A few of the other strong continuum sources (HL Tau, DG Tau, 
L1551 IRS5) are still surrounded by large remnant envelopes (Cabrit et al. 1996; Kitamura et al. 1996; Fridlund et al. 2002).

\subsection{Comparison of the Measured Masses and Theoretical Tracks}

The dynamically determined masses presented in this study can be used to test evolutionary model predictions. Hillenbrand \& White (2004) and Mathieu et al. (2007) present this type of assessment at a much more comprehensive level, including all young stars with accurately determined masses and a variety of evolutionary models. For our new stars we only present comparisons with one set of evolutionary model calculations, those of Baraffe et al. (1998), which are generally consistent with available constraints. Figure 9 shows a comparison of our results with the evolutionary tracks. We plot the tracks relative to the distance independent quantity $L / M^{2}$ (e.g. Simon et al. 2000). We used the $L_{\text {star }}$ values (Table 1, Column 5) computed by White \& Ghez (2001) and White \& Hillenbrand (2004), $T_{\text {eff }}$ from the Table 1, and dynamical masses derived from the disk rotation (Table 5). We show the location of GO Tau, Haro 6-13, and IRAS 04385+2550 (the sources for which we have clearly defined $1 \sigma$ stellar mass uncertainties) relative to the evolutionary tracks. We propagated the dynamical mass uncertainties through to the $L / M^{2}$ values and assumed \pm 0.5 spectral subtype uncertainties on $T_{\text {eff }}$.

Fortuitously, GO Tau, Haro 6-13 and IRAS 04385+2550 all have essentially the same spectral type (M0 or M0.5) and consequently the evolutionary models predict a stellar mass of about $0.7 \mathrm{M}_{\odot}$ for these stars (Figure 9$)$. The dynamical mass of IRAS $04385+2550(0.5 \pm$ $\left.0.1 \mathrm{M}_{\odot}\right)$ is smaller than its predicted mass by $\sim 2 \sigma$. Likewise, the dynamical mass of GO Tau $\left(0.61 \pm 0.02 \mathrm{M}_{\odot}\right)$ is $\sim 3 \sigma$ smaller than its predicted value. A systematic shift in temperature of 200-300 K cooler, caused by uncertainties in the temperature scale, could perhaps reconcile the discrepancy for these two stars. Alternatively, if GO Tau and IRAS 04385+2550 were located at distances 15-20\% farther away than 140 pc, it could also bring the dynamical masses more in line with the evolutionary tracks. Based on precise parallax measurements, Loinard (2008) found that the Taurus region spans a depth of $\sim 30 \mathrm{pc}$ (with individual distances of four stars ranging from 130-160 pc). Bertout \& Genova (2006) also find a similar spread of $\sim 30 \mathrm{pc}$ in the fullwidth of the distance distribution based on kinematic properties of Taurus moving group members with known radial velocities. Therefore, the uncertainties in the distance to individual stars in the Taurus region could potentially account for some of the discrepancy between the predicted and measured masses. A larger dynamical mass would also lower the vertical position of the stars toward older ages on the $L / M^{2} \mathrm{H}-\mathrm{R}$ diagram.

The dynamical mass of Haro $6-13\left(1.0 \pm 0.15 \mathrm{M}_{\odot}\right)$ is about $2 \sigma$ larger than the mass 
predicted from the evolutionary tracks. However, we note that the dynamical mass estimate suffers from potential systematic effects resulting from the limited number of velocity channels used in the $\mathrm{CO}$ analysis. Although the formal error on the inclination appears low, it is dependent on the assumed list of masked velocity channels. Lowering the inclination to about $30^{\circ}$ would bring the dynamical mass to $0.6 \mathrm{M}_{\odot}$, in better agreement with the evolutionary track for a very young object $(<1 \mathrm{Myr})$.

\section{Summary}

Our results indicate that it is difficult to find suitable PMS stars with masses below 0.5 $\mathrm{M}_{\odot}$ that have detectable circumstellar disks. Our findings are summarized below:

1) Based on our sample of 23 T Tauri stars with spectral types between K7-M7, we detected eight sources in $1.3 \mathrm{~mm}$ continuum emission but only four sources in CO line emission. We found no CO detections for stars with spectral types later than M1.

2) We combined our results with the larger sample of stars in the Taurus-Auriga star forming region that have been surveyed for millimeter continuum emission in the literature. We find a decrease in the continuum flux density from dusty disks around stars with spectral types M3-M9 compared with the sample of K0-M2 stars. This suggests that the masses of the circumstellar disks are decreasing with spectral type over the limited spectral range of K-M stars.

3) When they are detected, the CO gas disks around low-mass PMS stars can be sufficiently extensive that they are resolvable with the current generation of interferometers, providing a means to map the rotational dynamics of the disks.

4) We computed dynamical stellar masses of the four stars with CO line detections by mapping the rotation of their circumstellar disks. Unfortunately, obscuration from the parent molecular cloud presents a potential barrier to accurately mapping the rotation and measuring the stellar masses. A possible solution to recover the centrally obscured velocity channels would be to observe in the ${ }^{13} \mathrm{CO}$ or $\mathrm{C}^{18} 0$ isotopomers where the optical depth in the ambient cloud is smaller. Since the disks are expected to be even smaller in ${ }^{13} \mathrm{CO}$ than in ${ }^{12} \mathrm{CO}$ (Dartois et al. 2003), higher angular resolution and better sensitivity would be required to obtain more reliable mass estimates. The development of the Atacama Large Millimeter/submillimeter Array will greatly improve our ability to detect circumstellar disks around the lowest mass stars and brown dwarfs.

We thank T. Forveille, J. Kastner and B. Zuckerman for providing us with the inter- 
ferometric observations of GO Tau. We acknowledge all of the Plateau de Bure IRAM staff for their help during the observations. We also thank the anonymous referee for valuable comments that improved the manuscript. This research was carried out with partial support from NSF Grants 02-05427 and 06-07612. The observations were carried out with the IRAM Plateau de Bure Interferometer. IRAM is supported by INSU/CNRS (France), MPG (Germany), and IGN (Spain). This research has made use of the SIMBAD database, operated at CDS, Strasbourg, France

\section{A. Appendix}

\section{High Dispersion Optical Spectra of LkH $\alpha$ 358, IRAS 04108+2910 and IRAS $04216+2603$}

High dispersion $(\mathrm{R}=34,000)$ optical $(6330$ - $8750 \AA)$ spectra of IRAS $04108+2910$, IRAS 04216+2603 (2003 October 5), and $\mathrm{LkH} \alpha 358$ (2003 October 6) were obtained using the High-Resolution Echelle Spectrometer (HIRES; Vogt et al. 1994) on the W. M. Keck Telescope. The observational setup, data reduction, and spectral analysis are identical to that described in White \& Hillenbrand (2004). From these observations we measured spectral types, radial velocities, projected rotational velocities $(v \sin i)$, veiling at $6500 \AA$ and $8400 \AA\left(r_{6500}, r_{8400}\right)$, and equivalent widths of Li I 6708 and $\mathrm{H} \alpha$. These values are listed in Table 7. Portions of the extracted spectra are shown in Figure 10. All three stars have veiled spectra and broad $\mathrm{H} \alpha$ emission, indicative of accretion (e.g. White \& Basri 2003).

Prominent atomic and molecular features in the optical spectra of IRAS 04108+2910 and IRAS 04216+2603 indicate early M spectral types (M3 \pm 0.5 and M2.5 \pm 0.5 , respectively) that are rather well constrained. These spectral types are somewhat cooler than the M0 spectral type assigned to both of them, based on low resolution optical spectra presented in Kenyon et al. (1998). This is likely a consequence of veiling present in the spectra, which when unaccounted for, will diminish the depths of spectral features (e.g. TiO bands), and give the appearance of a hotter spectral type. The situation with $\mathrm{LkH} \alpha 358$ is more complicated. As noted in the text, $\mathrm{LkH} \alpha 358$ has been assigned a mid-M spectral type based on low resolution optical spectra in Cohen \& Kuhi (1979, M5.5) and Kenyon et al. (1998, M5-M6), but a much hotter spectral type (K7-M0) based on high resolution optical spectra in Muzerolle et al. (2003). Our new spectrum is consistent with the hotter spectral type (K7士1). The reason for the dramatic discrepancy in spectral type is unclear. Although $\mathrm{LkH} \alpha 358$ shows a modest amount of veiling, as noted above this typically shifts spectral types assigned with low resolution spectra toward hotter types, not cooler types. Private communication with authors S. Kenyon and J. Muzerolle confirm, to the best of their ability, that indeed 
$\mathrm{LkH} \alpha 358$ was observed in all cases. It seems unlikely that the photosphere of $\mathrm{LkH} \alpha 358$ has undergone an increase in temperature of nearly $1000 \mathrm{~K}$; while time variable features such as star spots can change the apparent temperature, these variations are expected to be less than a few hundred degrees. Other explanations such as a highly variable companion in combination with variable extinction to the primary seem more plausible.

\section{REFERENCES}

Andrews, S. M., Liu, M. C., Williams, J. P. \& Allers K. N. ApJ, 2008, 685, 1039

Andrews, S.M. \& Williams, J.P. 2005, ApJ, 631, 1134

Andrews, S.M. \& Williams, J.P. 2007, ApJ, 659, 705

Andersen, J. 1991, A\&A Rev., 3, 91

Baraffe, I., Chabrier, G., Allard, F., \& Hauschildt, P. H. 1998, A\&A, 337, 403

Beckwith, S. V. W., Sargent, A. I., Chini, R. S., \& Güsten, R. 1990, AJ, 99, 3

Bertout, C. \& Genova, F. A\&A, 460, 499

Briceño, C., Calvet, N., Kenyon, S., \& Hartmann, L. 1999, AJ, 118, 1354

Briceño, C., Calvet, N., Gomez, M., Hartmann, L. W., Kenyon, S. J. \& Whitney, B. A. 1993, PASP, 105, 686

Briceño, C., Hartmann, L., Stauffer, J., Martín, E. 1998, AJ, 115, 2074

Briceño, C., Luhman, K. L., Hartmann, L., Stauffer, J. R., \& Kirkpatrick, J. D. 2002, ApJ, 580,317

Cohen, M., \& Kuhi, L.V. 1979, ApJS, 41, 743

Cutri, R. M., et al. 2003, The IRSA 2MASS All-Sky Point Source Catalog (Pasadena, CA: NASA/IPAC)

Cabrit, S., Guilloteau, S., André, P., Bertout, C., Montmerle, T., \& Schuster, K. 1996, A\&A, 305,527

Connelley, M. S., Reipurth, B., \& Tokunaga, A. T. 2008, AJ, 135, 2496

Dartois, E., Dutrey, A., \& Guilloteau, S. 2003, A\&A, 399, 773 
Duchêne, G., Bontemps, S., Bouvier, J., André, P., Djupvik, A. A., \& Ghez, A. M. 2007, A\&A, 476, 229

Duchêne, G., Bouvier, J., Bontemps, S., André, P., \& Motte, F. 2004, A\&A, 427, 651

Dutrey, A., Guilloteau, S., Duvert, G., Prato, L. Simon, M., Schuster, K., \& Ménard, F. 1996, A\&A, 309, 493

Dutrey, A., Guilloteau, S., \& Simon, M. 1994, A\&A, 286, 149

Dutrey, A. Guilloteau, S., \&, Simon, M. 2003, A\&A, 402, 1003

Dutrey, A., et al. 2008, A\&A, 490, L15

Duvert, G., Dutrey, A., Guilloteau, S., Ménard, F. Schuster, K., Prato, L., \& Simon, M. 1998, A\&A, 332, 867

Fridlund, C. V. M., Bergman, P., White, G. J., Pilbratt, G. L., Tauber, J. A., 2002, A\&A, 382,573

Ghez, A. M., Neugebauer, G., \& Matthews, K. 1993, AJ, 106, 2005

Guilloteau, S. \& Dutrey, A. 1998, A\&A, 339, 467

Guilloteau, S., Dutrey, A., Pety, J., \& Gueth, F. 2008, A\&A, 478, L31

Hartigan, P. \& Kenyon, S. J. 2003, ApJ, 583, 334

Hartigan, P., Strom, K. M., \& Strom, S. E. 1994, ApJ, 427, 961

Hillenbrand, L. A. \& White, R. J. 2004, ApJ, 604, 741

Irwin, J., et al. 2007, MNRAS, 380, 541

Kenyon, S. J., Brown, D. I., Tout, C. A., \& Berlind, P. 1998, AJ, 115, 2491

Kenyon, S. J., Dobrzycka, D. \& Hartmann, L. 1994, AJ, 108, 1872

Kenyon, S. J. \& Hartmann, L. 1995, ApJS, 101, 117

Kitamura, Y., Kawabe, R., \& Saito, M. 1996, ApJ, 457, 277

Koerner, D.W. \& Sargent, A.I. 1995, AJ, 109, 2138

Konopacky, Q. M., Ghez, A. M., Rice, E. L., \& Duchêne, G. 2007, ApJ, 663, 394 
Kraus, A. L. \& Hillenbrand, L. A., 2007, AJ, 134, 2340

Kraus, A. L., White, R. J., \& Hillenbrand, L. A. 2006, ApJ, 649, 306

Loinard, L., Torres, R. M., Mioduszewski, A. J., \& Rodrguez, L. F. 2008, Rev. Mex. AA Ser. Conf., 34, 14

Luhman, K. L. 2000, ApJ, 544, 1044

Luhman, K. L. 2004, ApJ, 617, 1216

Luhman, K. L., Briceño, C. Stauffer, J. R., Hartmann, L., Barrado y Navascués, \& Caldwell, N. 2003a, ApJ, 590, 348

Luhman, K. L., Stauffer, J. R., Muench, A. A., Reike, G. H., Lada, E. A., Bouvier, J., \& Lada, C. J. 2003b, ApJ, 593, 1093

Martín, E. L. 2000, AJ, 120, 2114

Martín, E. L., Dougados, C., Magnier, E., Ménard, F., Magazzù, A., Cuillandre, J.-C., \& Delfosse, X. 2001, ApJ, 561, L195

Mathieu, R.D., Baraffe, I., Simon, M., Stassun, K.G. \& White, R. 2007, in Protostars and Planets V, eds. B. Reipurth, D. Jewitt, \& K. Keil (Tuscon: Univ. Arizona Press), 411

Motte, F. \& André, P. 2001, A\&A, 365, 440

Muzerolle, J., Hillenbrand, L., Calvet, N., Briceño, C. \& Hartmann, L. 2003, ApJ, 592, 266

Osterloh, M. \& Beckwith, S. V. W. 1995, ApJ, 439, 288

Pety, J., Gueth, F., Guilloteau, S., \& Dutrey, A., 2006, A\&A, 458, 841

Piétu, V., Dutrey, A., Guilloteau, S., Chapillon, E., \& Pety, J. 2006, A\&A, 460, L43

Piétu, V., Dutrey, A., \& Guilloteau, S. 2007, A\&A, 467, 163

Scholz, A., Jayawardhana, R., \& Wood, K., 2006, ApJ, 645, 1498

Simon, M., Dutrey, A., \& Guilloteau, S. 2000, ApJ, 545, 1034

Simon, M. 2001 in IAU Symp. 200, The Formation of Binary Stars, ed. H. Zinnecker \& R. D. Mathieu (San Francisco: ASP), 454

Simon, M., et al. 1995, ApJ, 443, 625 
Skrutskie, M. F., et al. 2006, AJ, 131, 1163

Stassun, K. G., Mathieu, R. D. \& Valenti, J. A. 2006, Nature, 440, 331

Vogt, S. S., et al. 1994, SPIE, 2198, 362

White, R. J. \& Basri, G. 2003, ApJ, 582, 1109

White, R. J. \& Ghez, A. M. 2001, ApJ, 556, 265

White, R. J. \& Hillenbrand, L. A. 2004, ApJ, 616, 998

Yokogawa, S., Kitamura, Y., Momose, M., \& Kawabe, R. 2002, in Proc. IAU 8th Asia-Pacific Regional Meeting, Vol. II, ed. S. Ikeuchi, J. Hearnshaw, \& T. Hanawa (Tokyo: ASJ), 239 
Table 1. Stellar Properties of the Sample

\begin{tabular}{|c|c|c|c|c|c|c|c|c|}
\hline $\begin{array}{c}\text { Name } \\
(1)\end{array}$ & $\begin{array}{l}\text { SpT } \\
(2)\end{array}$ & $\begin{array}{l}\text { Ref } \\
(3)\end{array}$ & $\begin{array}{l}\mathrm{L}_{\mathrm{J}} \\
\mathrm{L}_{\odot} \\
(4)\end{array}$ & $\begin{array}{c}\mathrm{L}_{\text {star }} \\
\mathrm{L}_{\odot} \\
(5)\end{array}$ & $\begin{array}{c}\mathrm{L}_{\text {bol }} \\
\mathrm{L}_{\odot} \\
(6)\end{array}$ & $\begin{array}{c}\mathrm{L}_{\text {bol }} \\
\mathrm{L}_{\odot} \\
(7)\end{array}$ & $\begin{array}{l}\mathrm{T}_{\text {eff }^{\mathrm{a}}} \\
(\mathrm{K}) \\
(8)\end{array}$ & Observation Dates \\
\hline $\mathrm{LkH} \alpha 358$ & K7 & 1 & $\ldots$ & 0.088 & 0.59 & 0.59 & 4060 & 2002 Oct 4,2002 Dec 20 \\
\hline GO Tau & M0 & 2 & 0.28 & 0.366 & & 0.37 & 3850 & 1995 Nov 22, 1995 Dec 5, 1997 Apr 5 \\
\hline Haro 6-13 & M0 & 3 & $\ldots$ & 2.11 & $\ldots$ & $\ldots$ & 3850 & 2002 Oct 4,2002 Dec 20 \\
\hline DN Tau & M0 & 3 & 0.91 & 0.68 & 1.0 & & 3850 & 2002 Oct 4 \\
\hline IRAS $04108+2803 \mathrm{~A}$ & M0 & 4 & $\ldots$ & & $\ldots$ & & 3850 & 2002 Oct 4 \\
\hline IRAS $04385+2550$ & M0.5 & 3 & $\ldots$ & 0.76 & & 0.18 & 3778 & 2002 Oct 4,2002 Dec 20 \\
\hline IQ Tau & M0.5 & 2 & 0.65 & 0.719 & 0.88 & & 3778 & 2002 Oct 4 \\
\hline CIDA-7 & M2-M3 & 5 & $\ldots$ & & & $\ldots$ & 3560 & 2002 Oct 4 \\
\hline IRAS $04216+2603$ & M2.5 & 1 & $\ldots$ & & & & 3488 & 2002 Oct 4 \\
\hline IRAS $04108+2910$ & M3 & 1 & $\ldots$ & $\ldots$ & & & 3415 & 2002 Oct 4 \\
\hline ITG $33 \mathrm{~A}$ & M3 & 6 & & & & 0.051 & 3415 & 2001 Nov 21,2002 Apr 15 \\
\hline CIDA-8 & M3.5 & 7 & 0.26 & & 0.25 & & 3343 & 2001 Nov 21,2002 Apr 15, 2002 Apr 17 \\
\hline CIDA-11 & M3.5 & 7 & 0.22 & & 0.26 & & 3343 & 2001 Nov 21,2002 Apr 15,2002 Apr 17 \\
\hline CIDA-12 & M4 & 7 & 0.09 & & 0.097 & & 3270 & 2001 Nov 21, 2002 Apr 15, 2002 Apr 17 \\
\hline FP Tau & M4 & 2 & 0.33 & 0.312 & & & 3270 & 2001 Nov 21 \\
\hline RX J0446.7+2459 & M4 & 5 & & & 0.245 & & 3270 & 2001 Nov 21, 2002 Apr 15, 2002 Apr 17 \\
\hline FN Tau & M5 & 2 & 0.50 & & 0.60 & & 3125 & 2002 Oct 4 \\
\hline CIDA-14 & M5 & 5 & & & 0.251 & & 3125 & 2001 Nov 21, 2002 Apr 15, 2002 Apr 17 \\
\hline CIDA-1 & M5.5 & 8 & $\ldots$ & & $\ldots$ & $\ldots$ & 3058 & 2001 Nov 21 \\
\hline V410 Anon 13 & M5.75 & 7 & & & 0.039 & 0.048 & 3024 & 2001 Nov 21 \\
\hline GM Tau & M6.5 & 8 & $\ldots$ & & & 0.047 & 2935 & 2001 Nov 21, 2002 Apr 15 \\
\hline MHO-4 & M7 & 8 & & 0.080 & 0.048 & 0.059 & 2880 & 2001 Nov 21 \\
\hline MHO-5 & M7 & 8 & & 0.047 & 0.11 & 0.065 & 2880 & 2001 Nov 21 \\
\hline
\end{tabular}

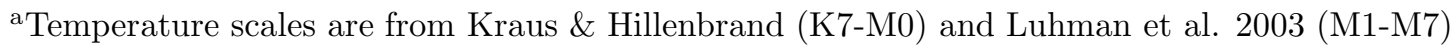

References. - (1) This paper; (2) Kenyon \& Hartmann 1995; (3) White \& Hillenbrand 2004; (4) Kenyon et al. 1998; (5) Briceño et al. 1999; (6) Martín 2000; (7) Briceño et al. 2002; (8) White \& Basri 2003 
Table 2. Millimeter Continuum Flux Densities

\begin{tabular}{|c|c|c|c|c|c|c|}
\hline Source & $\begin{array}{c}F_{2.6 \mathrm{~mm}}{ }^{\mathrm{a}} \\
(\mathrm{mJy})\end{array}$ & $\begin{array}{c}F_{1.3 \mathrm{~mm}}{ }^{\mathrm{a}} \\
(\mathrm{mJy})\end{array}$ & $\begin{array}{l}F_{1.3 \mathrm{~mm}}^{\mathrm{b}} \\
(\mathrm{mJy})\end{array}$ & $\begin{array}{l}F_{850 \mu \mathrm{m}^{\mathrm{b}}} \\
(\mathrm{mJy})\end{array}$ & $\alpha^{\mathrm{c}}$ & Ref \\
\hline $\mathrm{LkH} \alpha 358$ & $4.0 \pm 0.6$ & $17.1 \pm 1.0$ & $32 \pm 6$ & & $2.07 \pm 0.35$ & 1 \\
\hline GO Tau & $8.2 \pm 0.6$ & $53.1 \pm 2.6$ & $57 \pm 2^{\mathrm{d}}$ & $173 \pm 7$ & $2.73 \pm 0.07$ & 2,3 \\
\hline Haro 6-13 & $29.5 \pm 0.6$ & $105.2 \pm 1.5$ & $124 \pm 13$ & $395 \pm 56$ & $1.81 \pm 0.03$ & 4,3 \\
\hline DN Tau & $18.2 \pm 1.8$ & $88.8 \pm 6.8$ & $90 \pm 2^{\mathrm{e}}$ & $201 \pm 7$ & $2.07 \pm 0.08$ & 2,3 \\
\hline IRAS $04108+2803 \mathrm{~A}$ & $<5.1$ & $<19.5$ & $<20$ & & & 5 \\
\hline IRAS $04385+2550$ & $6.1 \pm 0.5$ & $24.4 \pm 0.9$ & $30 \pm 5$ & & $1.95 \pm 0.13$ & 5 \\
\hline IQ Tau & $12.5 \pm 1.7$ & $60 \pm 10$ & $87 \pm 11$ & $178 \pm 3$ & $2.32 \pm 0.11$ & 4,3 \\
\hline CIDA-7 & $<4.8$ & $<18.6$ & & $38 \pm 8$ & $>1.81$ & 3 \\
\hline IRAS $04216+2603$ & $<5.1$ & $<19.8$ & & & & \\
\hline IRAS $04108+2910$ & $<5.1$ & $<19.8$ & & & & \\
\hline ITG 33A & $<2.7$ & $<5.1$ & & & & \\
\hline CIDA-8 & $<2.1$ & $8.2 \pm 1.4$ & & $27 \pm 3$ & $2.96 \pm 0.51$ & 3 \\
\hline CIDA-11 & $<2.1$ & $<4.2$ & & $<8$ & & 3 \\
\hline CIDA-12 & $<2.1$ & $<4.5$ & & $<7$ & & 3 \\
\hline FP Tau & $<9.3$ & $<9.3$ & & & & \\
\hline RXJ0446.7+2459 & $<2.1$ & $<4.5$ & & & & \\
\hline FN Tau & $<4.8$ & $<17.7$ & $31 \pm 9$ & & $>2.37$ & 4 \\
\hline CIDA-14 & $<2.1$ & $<4.5$ & & & & \\
\hline CIDA-1 & $<7.8$ & $13.5 \pm 2.8$ & & & $>0.77$ & \\
\hline V410 Anon 13 & $<6.9$ & $<9.0$ & & & & \\
\hline GM Tau & $<2.4$ & $<4.8$ & & & & \\
\hline MHO-4 & $<7.8$ & $<9.6$ & & & & \\
\hline MHO-5 & $<7.8$ & $<9.0$ & & & & \\
\hline
\end{tabular}

${ }^{a}$ Continuum flux densities presented in this paper.

${ }^{\mathrm{b}}$ Continuum flux densities from other works cited in the last column.

${ }^{\mathrm{c}}$ The spectral index $\alpha$ is calculated from all available flux densities listed in the Table.

${ }^{\mathrm{d}}$ For GO Tau, Beckwith et al. 1990 also measured a flux density of $83 \pm 12 \mathrm{mJy}$ at $1.25 \mathrm{~mm}$. 
${ }^{\text {e}}$ For DN Tau, Beckwith et al. 1990 also measured a flux density of $84 \pm 13$ mJy at $1.25 \mathrm{~mm}$.

References. - (1) Osterloh \& Beckwith 1995 (1.3 mm); (2) Andrews \& Williams 2007 (1.33 mm); (3) Andrews \& Williams 2005 (0.863 mm); (4) Beckwith et al. 1990 (1.25 mm); (5) Motte \& André 2001 (1.3 mm) 
Table 3. Measured Millimeter Continuum Source Coordinates (J2000.0)

\begin{tabular}{llll}
\hline \hline \multicolumn{1}{c}{ Name } & \multicolumn{1}{c}{ RA } & \multicolumn{1}{c}{$\begin{array}{c}\text { Dec } \\
(\mathrm{h} \mathrm{m} \mathrm{s})\end{array}$} & \multicolumn{1}{c}{$\left.\begin{array}{l}\sigma_{\text {pos }} \\
\left({ }^{\prime} \prime\right.\end{array}\right)$}
\end{tabular}

Table 4. Continuum Dust Disk Parameters (d=140 pc)

\begin{tabular}{lll}
\hline \hline & $\begin{array}{c}R_{\text {out }} \\
\mathrm{AU}\end{array}$ & $\begin{array}{c}M_{\text {dust }} \\
\mathrm{M}_{\odot}\end{array}$ \\
\hline LkH $\alpha$ 358 & $60 \pm 20$ & 0.0015 \\
GO Tau & $70 \pm 20$ & 0.007 \\
Haro 6-13 & $90 \pm 10$ & 0.030 \\
DN Tau & $>30$ & $>0.015$ \\
IRAS 04385+2550 & $>16$ & $>0.004$ \\
IQ Tau & $>25$ & $>0.010$ \\
CIDA-8 & $>9$ & $>0.0013$ \\
CIDA-1 & $>12$ & $>0.002$ \\
\hline
\end{tabular}

Note. - The dust masses are uncertain by at least a factor of 2 (see Section 3.1). 
Table 5. Geometric and Dynamic Parameters of the CO Disks (d=140 pc)

\begin{tabular}{lcccccc}
\hline \hline \multicolumn{1}{c}{ Name } & $\begin{array}{c}P A_{\mathrm{CO}} \\
\left({ }^{\circ}\right)\end{array}$ & $\begin{array}{c}i_{\mathrm{CO}} \\
\left({ }^{\circ}\right)\end{array}$ & $\begin{array}{c}R_{\text {out }} \\
(\mathrm{AU})\end{array}$ & $\begin{array}{c}V_{100} \sin i \\
\left(\mathrm{~km} \mathrm{~s}^{-1}\right)\end{array}$ & $v$ & $\begin{array}{c}M_{*} \\
\left(\mathrm{M}_{\odot}\right)\end{array}$ \\
\hline LkH $\alpha 358$ & $119 \pm 2$ & $28 \pm 9$ & $150 \pm 17$ & $1.35 \pm 0.04$ & $(0.5)$ & {$[0.5-2.0]$} \\
GO Tau & $114 \pm 1$ & $52 \pm 1$ & $920 \pm 20$ & $1.84 \pm 0.01$ & $0.50 \pm 0.02$ & $0.61 \pm 0.02$ \\
Haro 6-13 & $90 \pm 2$ & $40 \pm 3$ & $>180$ & $1.93 \pm 0.03$ & $0.49 \pm 0.02$ & $1.00 \pm 0.15$ \\
IRAS 04385+2550 & $52 \pm 1$ & $52 \pm 5$ & $300 \pm 10$ & $1.63 \pm 0.02$ & $0.51 \pm 0.02$ & $0.5 \pm 0.1$ \\
\hline
\end{tabular}

Note. - Parameters within parenthesis have been fixed to this value for the fit. For the masses, values within brackets indicate the range for $\pm 1 \sigma$ difference in the inclination.

Table 6. Physical Parameters of the CO Disks ( $\mathrm{d}=140 \mathrm{pc})$

\begin{tabular}{lcccccccc}
\hline \hline \multirow{1}{*}{ Name } & \multicolumn{3}{c}{ Hydrostatic Assumption $^{\mathrm{a}}$} & \multicolumn{5}{c}{ Free Scale Height } \\
& $T_{100}$ & $q$ & $H_{100}$ & $h$ & $T_{100}$ & $q$ & $H_{100}$ & $h$ \\
& $(\mathrm{~K})$ & & $(\mathrm{AU})$ & & $(\mathrm{K})$ & & $(\mathrm{AU})$ & \\
\multicolumn{1}{c}{$(1)$} & $(2)$ & $(3)$ & $(4)$ & $(5)$ & $(6)$ & $(7)$ & $(8)$ & $(9)$ \\
\hline LkH $\alpha$ 358 & $26 \pm 1$ & $(0.5)$ & 13 & -1.25 & & & & \\
GO Tau & $31 \pm 1$ & $0.49 \pm 0.02$ & 19 & -1.25 & $23 \pm 2$ & $0.35 \pm 0.04$ & $35 \pm 6$ & $-1.0 \pm 0.1$ \\
Haro 6-13 & $29 \pm 2$ & $0.35 \pm 0.07$ & 15 & -1.33 & & & & \\
IRAS 04385+2550 & $36 \pm 1$ & $0.56 \pm 0.02$ & 23 & -1.22 & $29 \pm 1$ & $0.30 \pm 0.03$ & $47 \pm 5$ & $-0.9 \pm 0.2$ \\
\hline
\end{tabular}

${ }^{a}$ In Cols 2-5, temperatures and scale heights are derived assuming hydrostatic equilibrium.

${ }^{\mathrm{b}}$ In Col 6-9, temperatures and scale heights are fitted independently with power laws. 
Table 7. Spectroscopic Properties

\begin{tabular}{llllllll}
\hline \hline \multicolumn{1}{c}{ Star } & \multicolumn{1}{c}{$\mathrm{SpT}$} & \multicolumn{1}{c}{$\mathrm{RV}$} & $\begin{array}{c}v \sin i \\
(\mathrm{~km} / \mathrm{s})\end{array}$ & \multicolumn{1}{c}{$r_{6500}$} & $r_{8400}$ & $\begin{array}{c}\mathrm{EW}[\mathrm{Li}]^{\mathrm{a}} \\
(\AA)\end{array}$ & $\begin{array}{c}\mathrm{EW}[\mathrm{H} \alpha]^{\mathrm{a}} \\
(\AA)\end{array}$ \\
\hline LkH $\alpha$ 358 & $\mathrm{K} 7 \pm 1$ & $21.4 \pm 1.2$ & $27 \pm 3$ & $0.2 \pm 0.2$ & $0.3 \pm 0.3$ & -43 & 0.48 \\
IRAS 04216+2603 & $\mathrm{M} 2.5 \pm 0.5$ & $17.6 \pm 0.3$ & $13.5 \pm 0.8$ & $0.7 \pm 0.1$ & $0.3 \pm 0.1$ & -19 & 0.38 \\
IRAS 04108+2910 & $\mathrm{M} 3 \pm 0.5$ & $18.8 \pm 0.3$ & $\leq 7$ & $2.0 \pm 0.1$ & $1.8 \pm 0.4$ & -128 & 0.33 \\
\hline
\end{tabular}

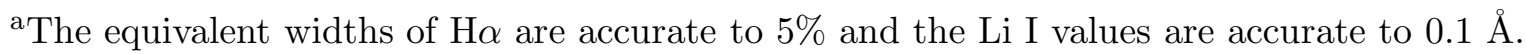




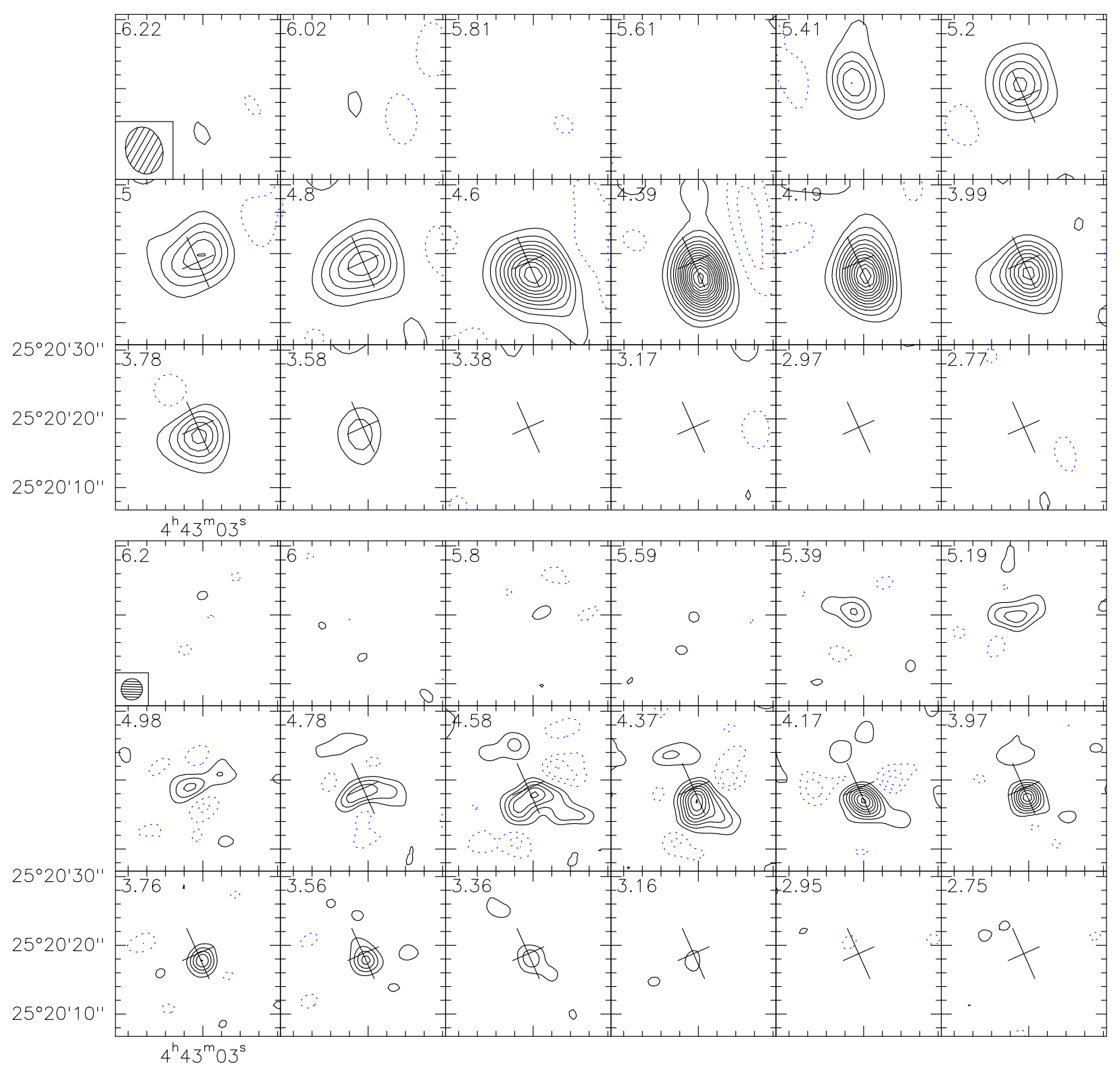

Fig. 1. $-{ }^{12} \mathrm{CO} \mathrm{J}=1-0$ and $\mathrm{J}=2-1$ maps for GO Tau, with LSR velocity indicated in each panel. The systemic velocity is $5.0 \mathrm{~km} \mathrm{~s}^{-1}$. The crosses indicate the disk orientation and center position; channels with no crosses have not been used in the analysis of the rotating disk. Top: $\mathrm{J}=1-0$ transition: resolution is $6.9 \times 5.4^{\prime \prime}$ at $\mathrm{PA} 15^{\circ}$, contour spacing $0.1 \mathrm{Jy}$, or $0.25 \mathrm{~K}(\sim 2.5 \sigma)$. Bottom: $\mathrm{J}=2-1$ transition: resolution is $3.1^{\prime \prime}$, contour spacing $0.3 \mathrm{Jy}$, or 0.7 $\mathrm{K}(\sim 2.5 \sigma)$. 


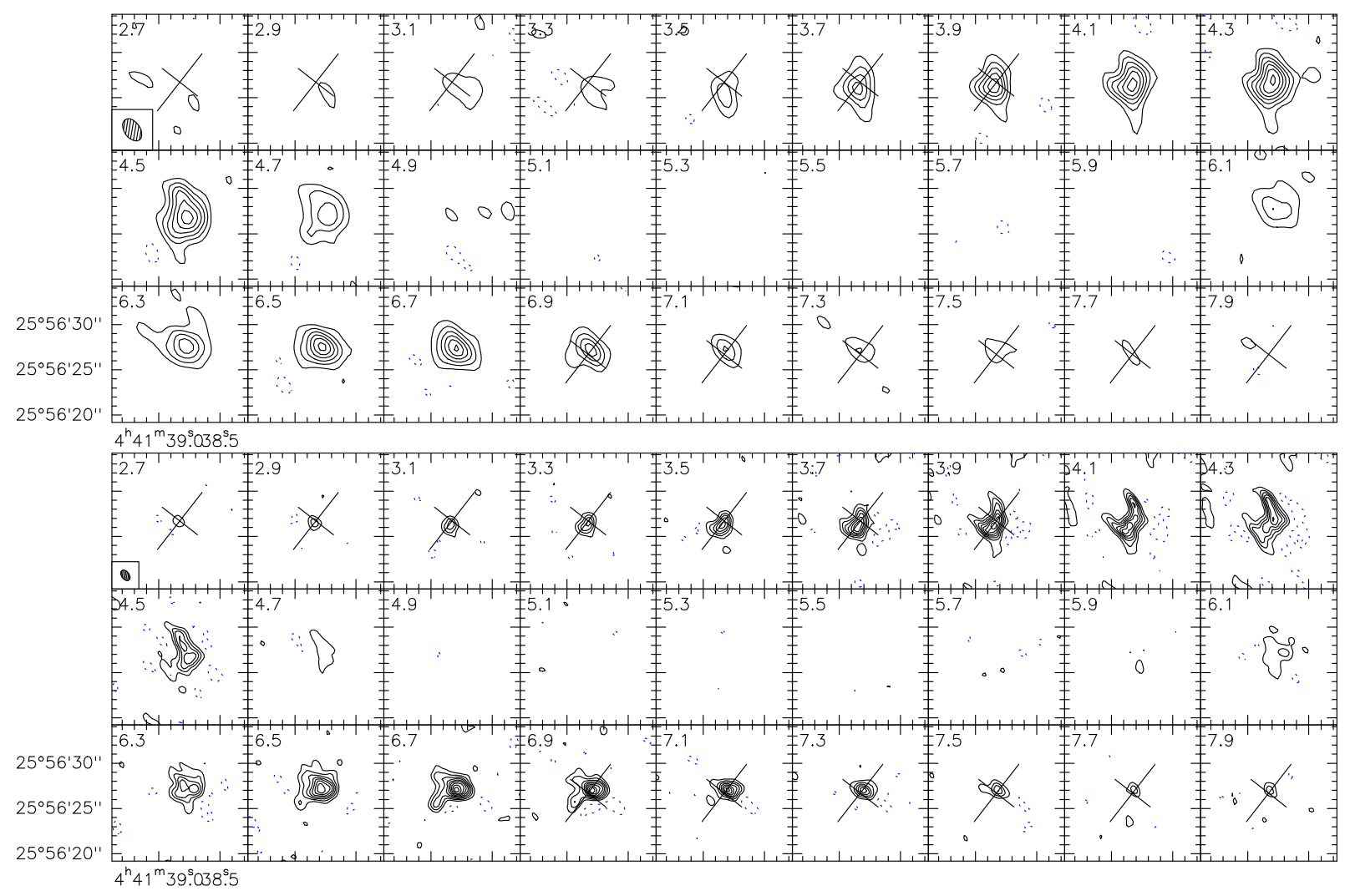

Fig. 2.- Same as Fig.1 for IRAS $04385+2550$. The systemic velocity is $5.30 \mathrm{~km} \mathrm{~s}^{-1}$. Top: $\mathrm{J}=1-0$ transition: resolution is $2.7 \times 1.7^{\prime \prime}$ at $\mathrm{PA} 36^{\circ}$, contour spacing $0.11 \mathrm{Jy}$, or $2.1 \mathrm{~K}(\sim 3 \sigma)$. Bottom: J=2-1 transition: resolution is $1.3 \times 0.8^{\prime \prime}$, contour spacing $0.13 \mathrm{Jy}$, or $2.8 \mathrm{~K}(\sim 3 \sigma)$.

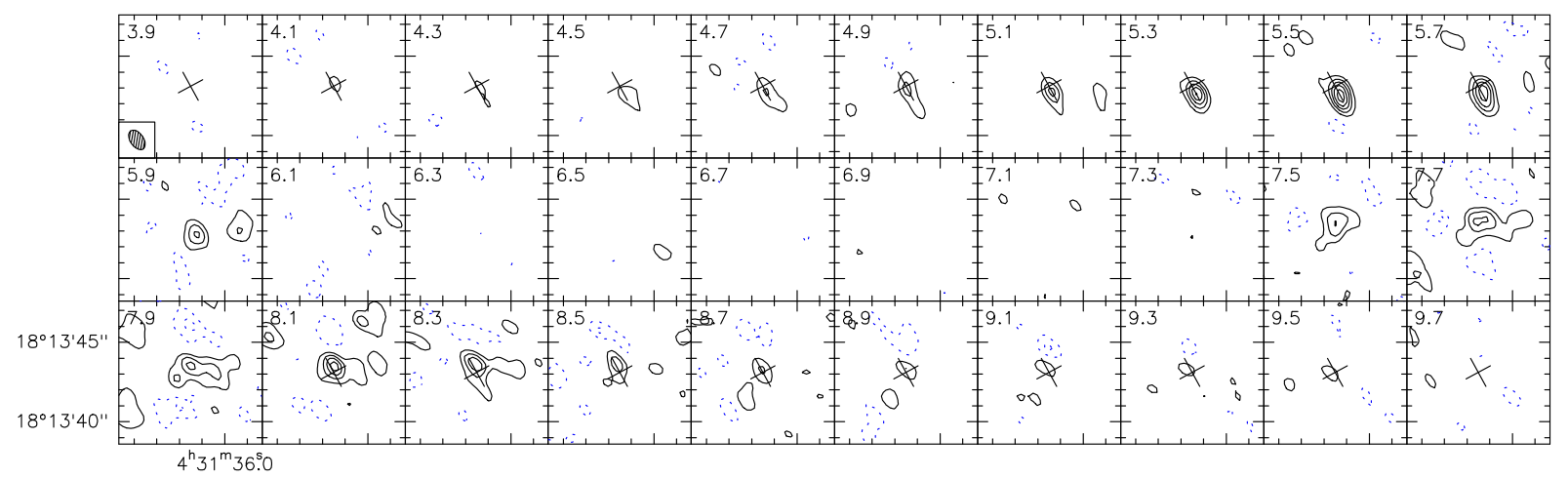

Fig. 3.- Same as Fig.1 for $\mathrm{LkH} \alpha$ 358. The systemic velocity is $6.80 \mathrm{~km} \mathrm{~s}^{-1}$. J $=2-1$ transition: resolution is $1.38 \times 0.83^{\prime \prime}$ at PA $35^{\circ}$, contour spacing $0.13 \mathrm{Jy}$, or $2.6 \mathrm{~K}(\sim 2.9 \sigma)$. 


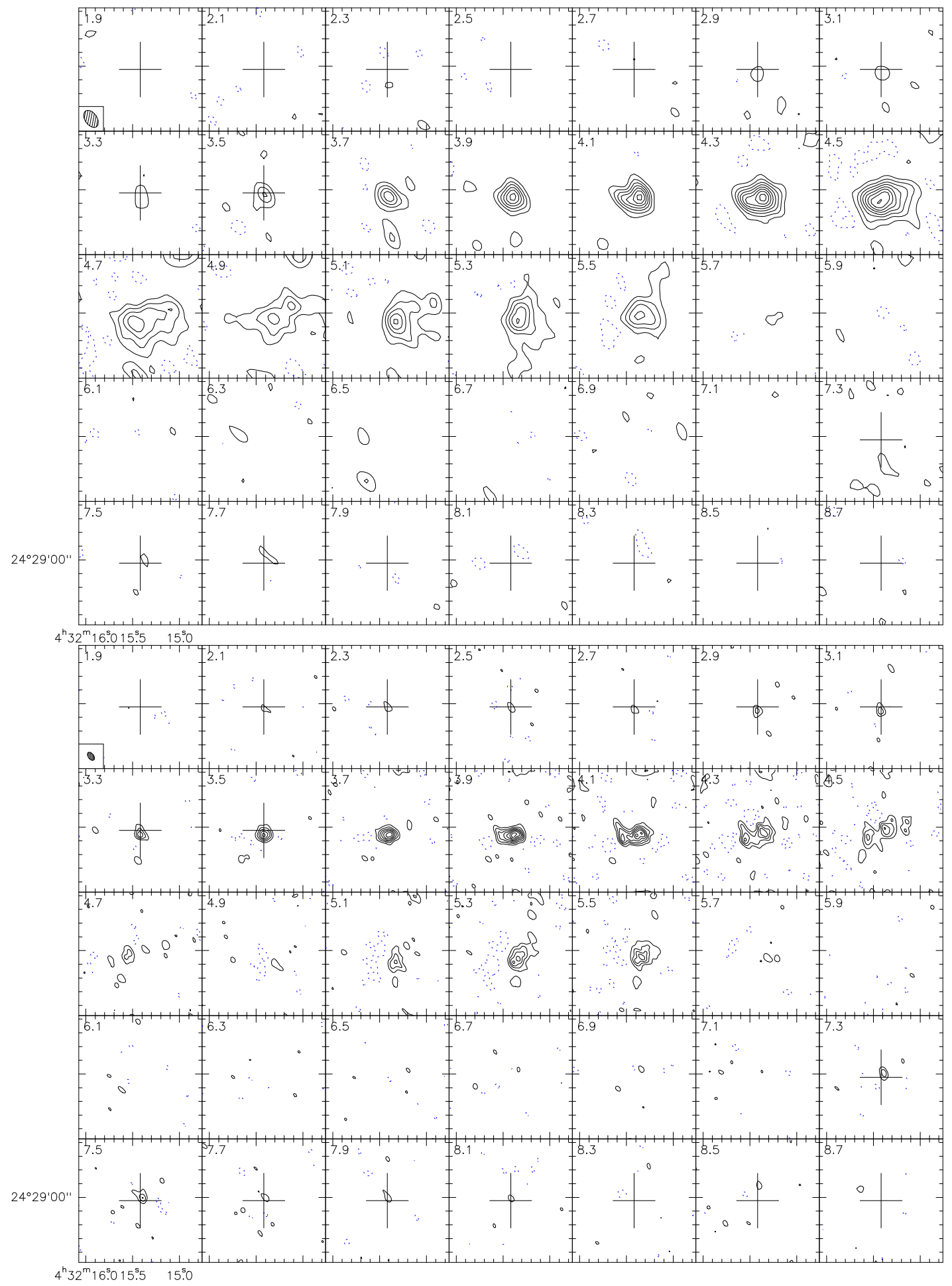

Fig. 4.- Same as Fig.1 for Haro 6-13. The systemic velocity is $5.10 \mathrm{~km} \mathrm{~s}^{-1}$. Top: J=1-0 transition: resolution is $2.8 \times 2.7^{\prime \prime}$ at PA $34^{\circ}$, contour spacing $0.10 \mathrm{Jy}$, or $1.9 \mathrm{~K}(\sim 2.8 \sigma)$. Bottom: $\mathrm{J}=2-1$ transition: resolution is $1.35 \times 0.80^{\prime \prime}$ at $\mathrm{PA} 37^{\circ}$, contour spacing $0.13 \mathrm{Jy}$, or $2.8 \mathrm{~K}(\sim 2.8 \sigma)$. 


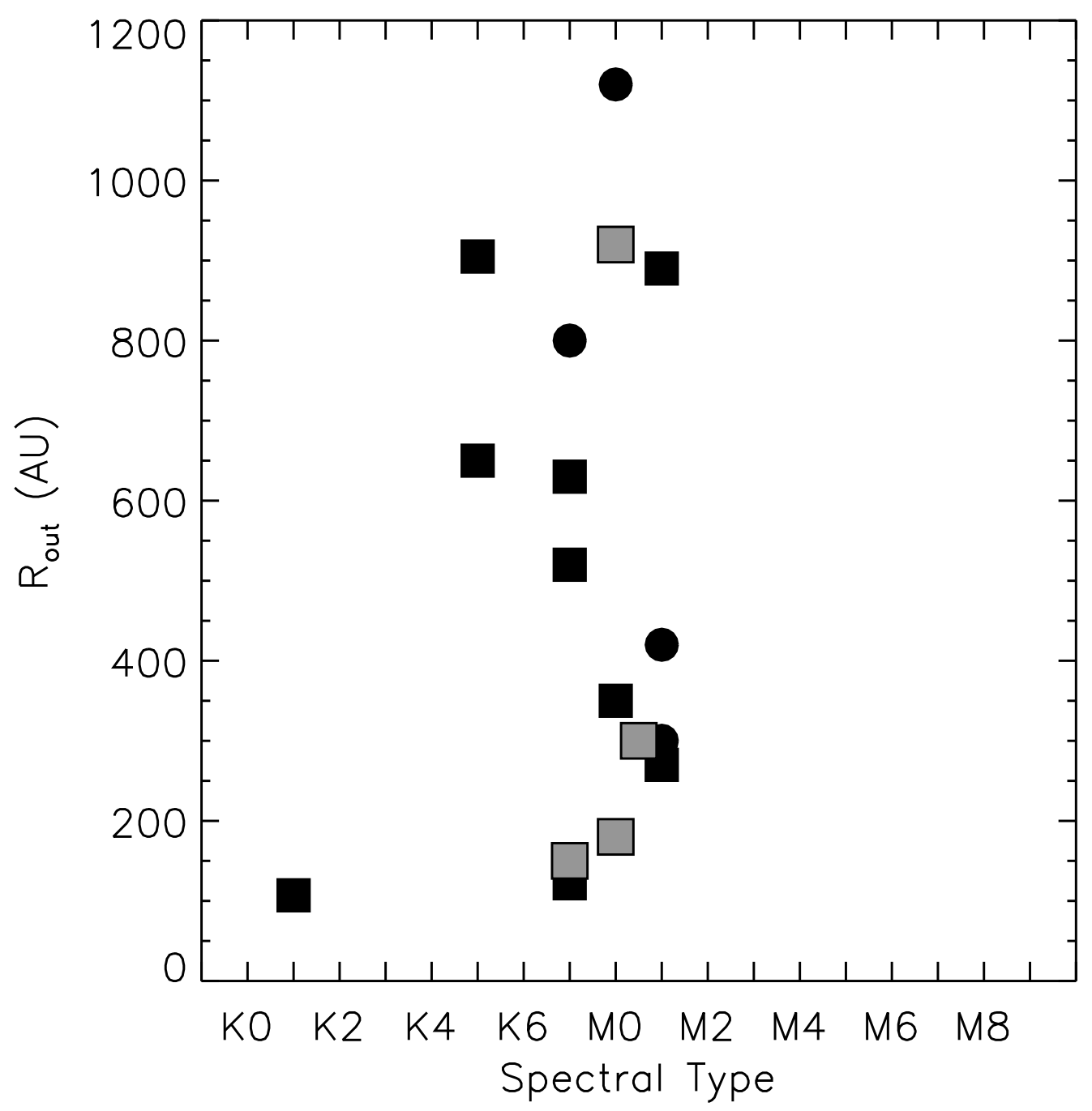

Fig. 5.- Radii of $\mathrm{CO}$ disks detected around $\mathrm{T}$ Tauri stars of spectral types K-M in the Taurus-Auriga star forming region. The large shaded squares represent our measurements of $R_{\text {out }}$ for LkH $\alpha 358$, GO Tau, Haro 6-13, and IRAS 04385+2550. The solid black squares represent values of $R_{\text {out }}$ measured for the single stars CY Tau, DL Tau (Simon et al. 2000), BP Tau (Dutrey et al. 2003), DM Tau, LkCa 15 (Piétu et al. 2007), and GM Aur (Dutrey et al. 2008), and the radius at half-maximum measured for DO Tau, RY Tau (Koerner \& Sargent 1995), and Haro 6-5B (Yokogawa et al. 2002). The solid black circles represent the radii of the circumbinary disks around GG Tau A (Dutrey et al. 1994), UY Aur (Duvert et al. 1998), UZ Tau E (Simon et al. 2000), and HH 30 (Pety et al. 2006; Guilloteau et al. 2008). 


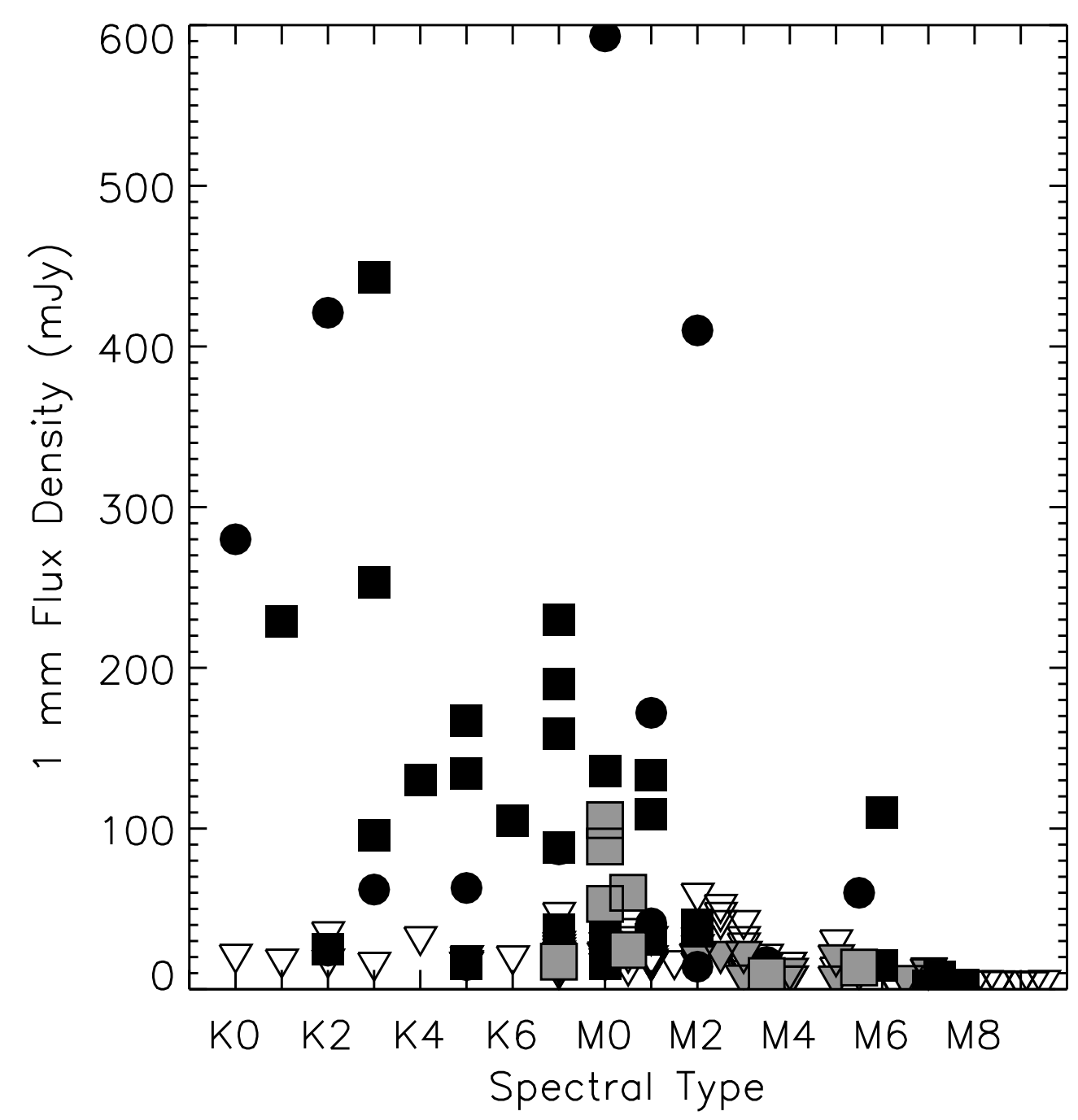

Fig. 6.- $1.3 \mathrm{~mm}$ flux density versus spectral type for K-M stars in the Taurus-Auriga star forming region. The sample is compiled from Beckwith et al. (1990), Osterloh \& Beckwith (1995), Motte \& André (2001), Scholz et al. (2006), and our sample of 23 low-mass PMS stars. The filled black squares and circles represent detections at $1.3 \mathrm{~mm}$ for single and binary sources, respectively, while the open triangles represent the $3 \sigma$ upper limits. The continuum flux densities that we measured in Table 2 are represented by the large shaded symbols. There are two stars (L1551 IRS5 and HL Tau) in the K2-K5 range that have continuum fluxes larger than $800 \mathrm{mJy}$. 

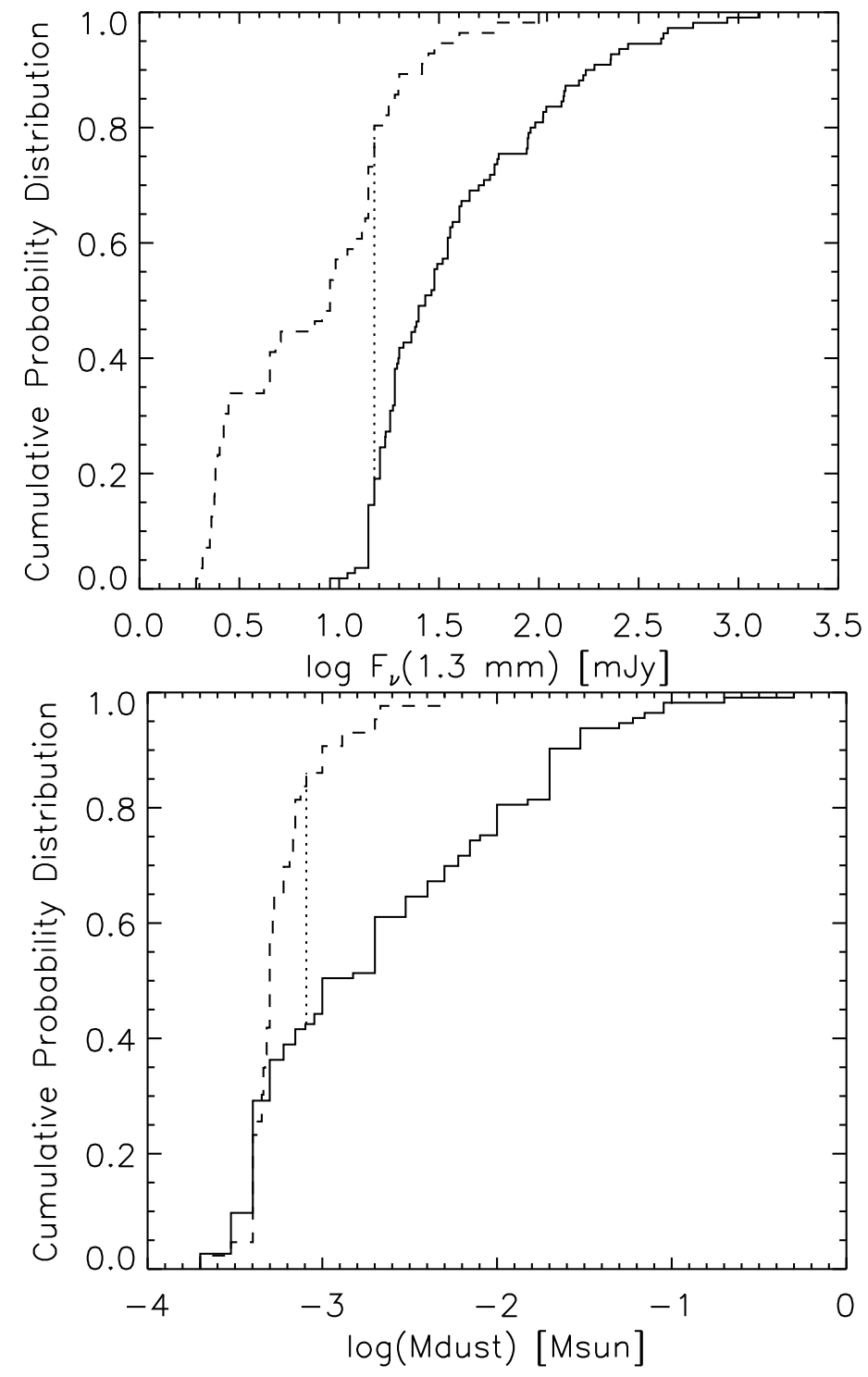

Fig. 7.- Top: Cumulative probability function for $1.3 \mathrm{~mm}$ continuum flux densities. The solid line represents the sample of $\mathrm{T}$ Tauri stars with spectral types between K0-M2, while the dashed line represents the sample between M3-M9. The maximum difference between the distributions of 0.61 (dotted line, Kolmogorov-Smirnov statistic) indicates that the distributions are significantly different. Bottom: Cumulative probability function for the values of $M_{\text {dust }}$. The samples are defined in the same way as the top panel. The maximum difference between the distributions of 0.44 indicates that the distributions are significantly different. In both cases, the Kolmogorov-Smirnov test indicates a negligible probability $(p \ll 0.01)$ that the two distributions are actually the same. 


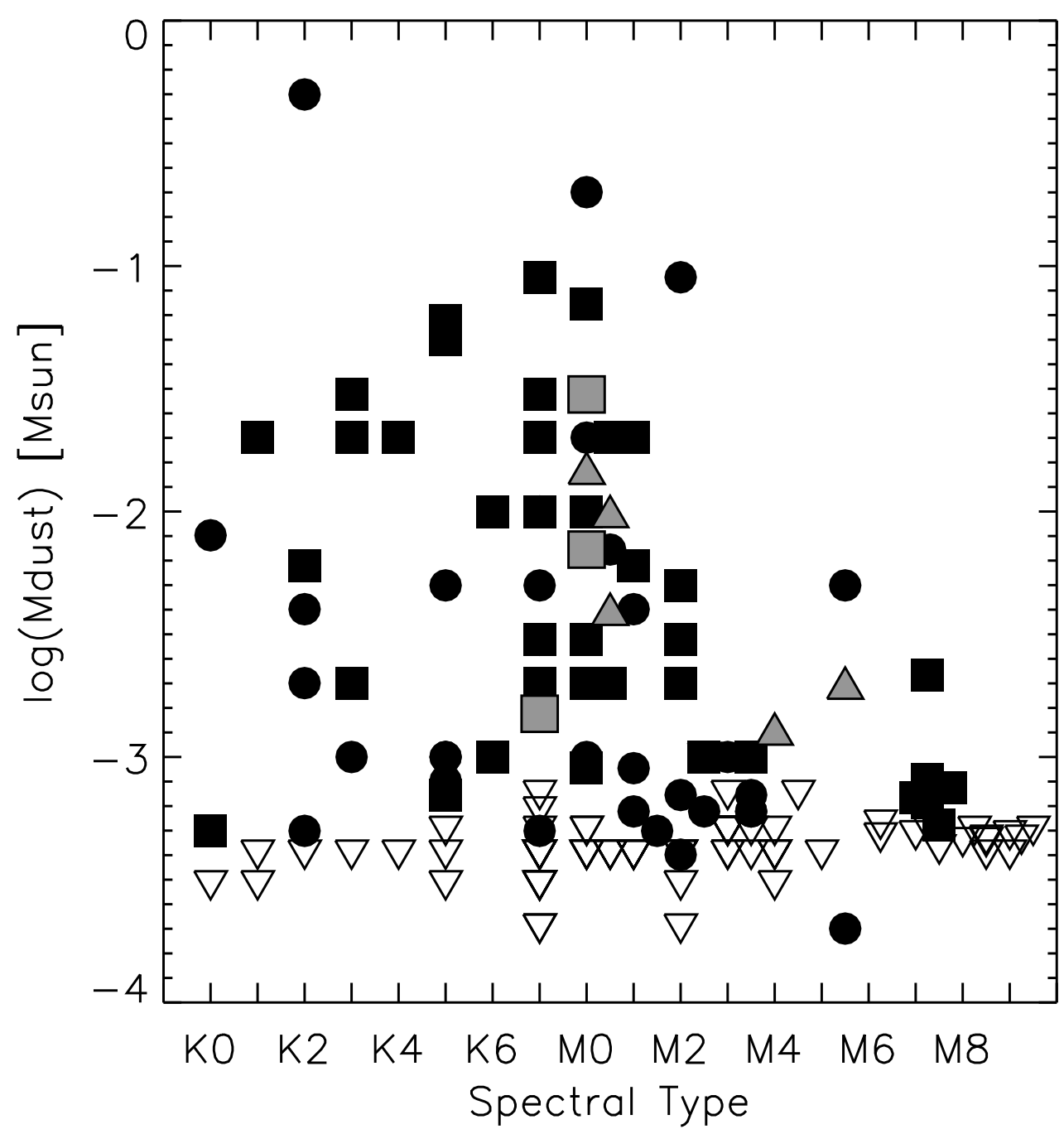

Fig. 8.- Dust disk masses versus spectral type for K-M stars in the Taurus-Auriga star forming region. The filled black squares and circles represent the dust masses determined by Andrews \& Williams (2005) and Scholz et al. (2006) for single and binary sources, respectively, while the open downward pointing triangles represent their $3 \sigma$ upper limits. Our dust disk mass measurements listed in Table 4 are represented by the large shaded squares while the shaded upward pointing triangles represent our lower limits. 


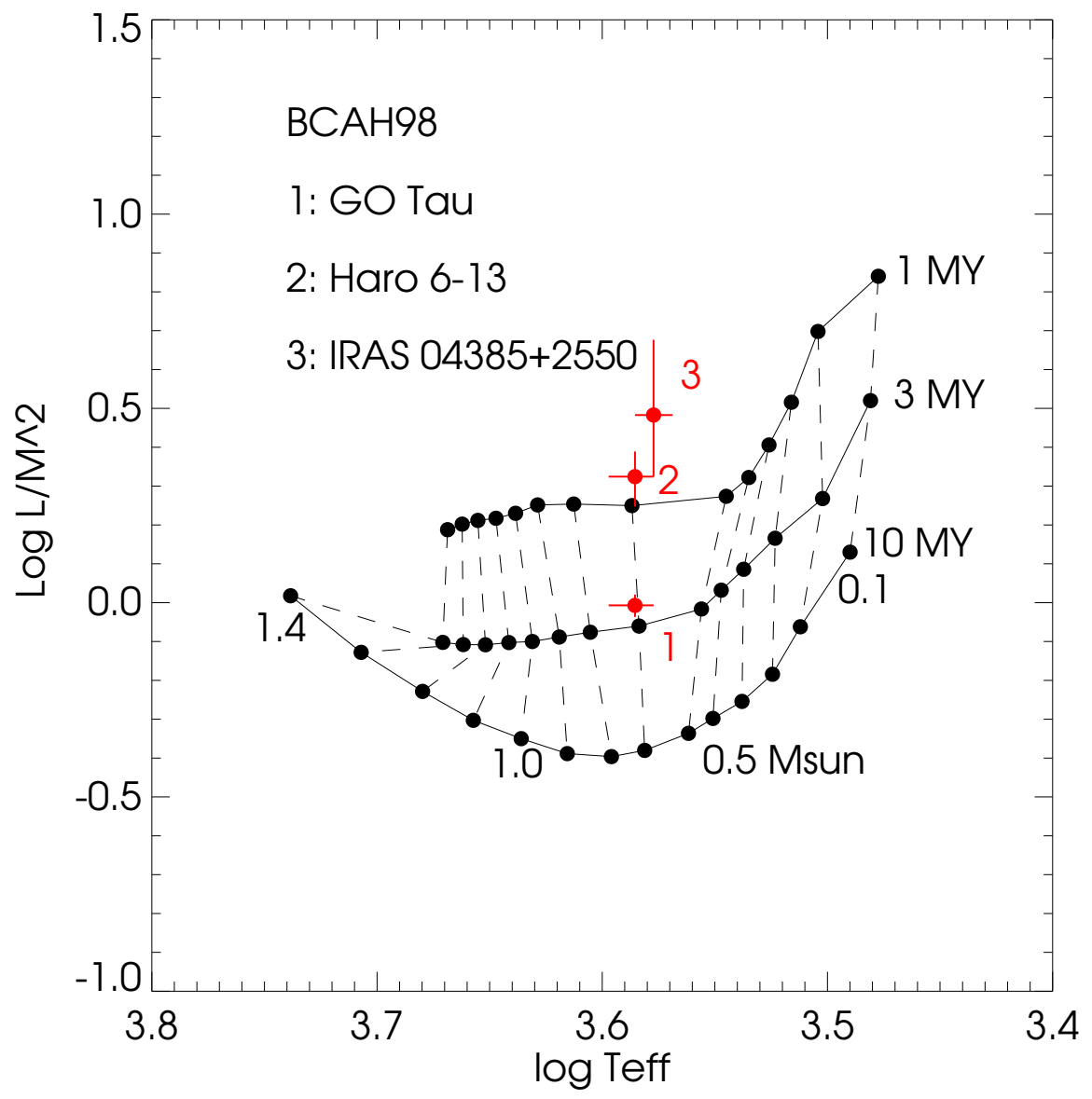

Fig. 9.- Evolutionary tracks computed by Baraffe et al. (1998) plotted as the distance independent quantity $L / M^{2}$ versus $T_{\text {eff }}$. The tracks are plotted from 0.1-1.4 $M_{\odot}$ at intervals $0.1 M_{\odot}$ for an age range of 1-10 Myr. We show the location of GO Tau, Haro 6-13, and

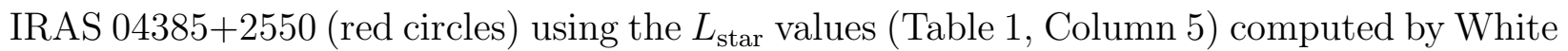
\& Ghez (2001) and White \& Hillenbrand (2004), $T_{\text {eff }}$ from Table 1, and the dynamical masses derived from the $\mathrm{CO}$ emission (Table 5). We propagated the dynamical mass uncertainties through to the $L / M^{2}$ values and assumed \pm 0.5 spectral subtype uncertainties on $T_{\text {eff }}$. 


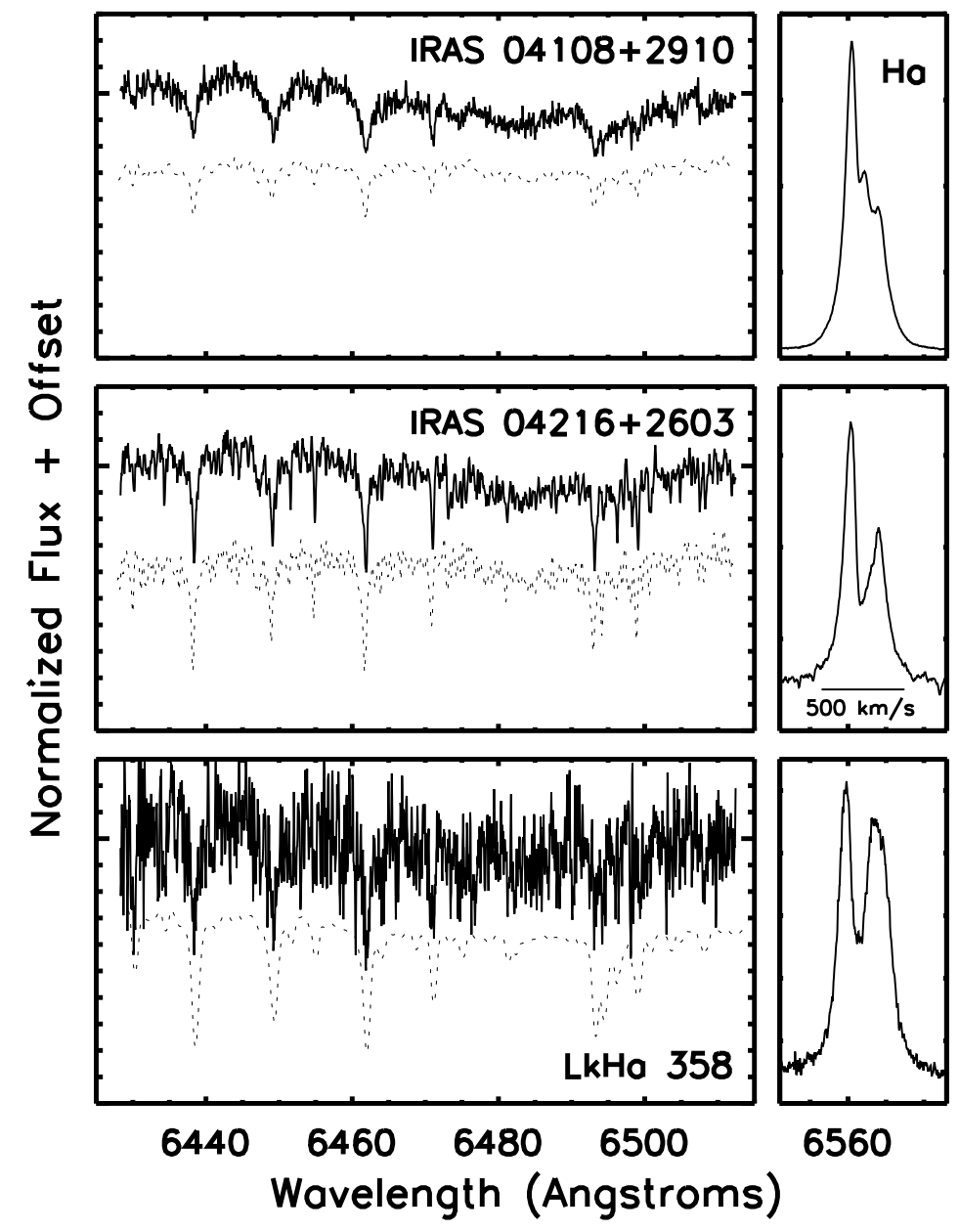

Fig. 10.- Portions of the Keck/HIRES spectra within the $\mathrm{R}$ band (left) and $\mathrm{H} \alpha$ emission profiles (right); both panels have the same wavelength scale. The best-fit dwarf standards, rotationally broadened and optically veiled, are shown as dashed lines for comparison. The strong, broad $\mathrm{H} \alpha$ emission profiles suggest that all three stars are accreting. 